DOI: $10.24850 / j-t y c a-2021-05-04$

Artículos

\title{
Análisis regional de frecuencia de sequías basado en L- momentos para la cuenca del río Bravo, México
}

\section{Regional drought frequency analysis based on L- moments for the Rio Grande River Basin, Mexico}

Jesús Alberto Ceballos-Tavares ${ }^{1}$, ORCID: https://orcid.org/0000-00026534-5515

David Ortega-Gaucin², ORCID: https://orcid.org/0000-0002-5336-7442

${ }^{1}$ Instituto Mexicano de Tecnología del Agua, Jiutepec, Morelos, México, jesus.ceballos@posgrado.imta.edu.mx

2Instituto Mexicano de Tecnología del Agua, Jiutepec, Morelos, México, dortega@tlaloc.imta.mx

Autor para correspondencia: David Ortega-Gaucin, dortega@tlaloc.imta.mx

\section{Resumen}


Se realizó el análisis regional de frecuencias basado en L-momentos (ARFLM) para determinar las características de los periodos de sequía meteorológica ocurridos en la cuenca del río Bravo durante el lapso 19842013. Para ello, se usaron registros de precipitación mensual de 90 estaciones climatológicas, que fueron sometidos a un proceso de control de calidad y homogeneización de datos. Mediante un proceso iterativo, se identificaron cinco regiones homogéneas $(\mathrm{RH})$ de acuerdo con el criterio de precipitación media anual. Las funciones de distribución de probabilidades que mejor se ajustaron a los registros fueron Gaucho (RH1) y Logística Generalizada (RH2 a RH5). Con base en estas funciones se determinaron los cuantiles (valores de precipitación anual asociados con un determinado valor de probabilidad de ocurrencia), y se generaron mapas de déficit de precipitación con periodos de retorno de 5, 10, 15, 20, 50 y 100 años. Los resultados indican que en la cuenca se presentan eventos de sequía meteorológica con duración promedio entre 1.7 y 2.5 años, y con una recurrencia media de 3.9 a 4.5 años; el periodo de sequía más prolongado ocurrió entre los años 1993 y 2002, y el periodo que presentó el mayor déficit de lluvia fue 2011-2012. Las regiones más afectadas se ubican en los estados de Chihuahua y Coahuila, donde los niveles de disminución de la precipitación pueden superar el $80 \%$ de déficit, lo cual indica que para un periodo de retorno de 100 años, más de la mitad de la cuenca presentaría un grado de sequía extraordinaria, lo cual tendría graves repercusiones en los distintos sectores socioeconómicos.

Palabras clave: sequía meteorológica, precipitación, análisis regional de frecuencias, L-momentos, cuenca del río Bravo. 


\section{Abstract}

In order to determine the characteristics of meteorological drought periods that occurred in the Rio Grande River Basin in the 1984-2013 period, a regional frecuency analysis based on L-moments (RFA-LM) was conducted. The analysis used precipitation records from 90 weather stations, which were subject of a quality control and data homogenization process. Through an iterative process, five homogeneous regions (HR) were identified according to the annual mean precipitation criterion. The probability distribution functions that best fitted the records were Gaucho (HR1) and Generalized Logistic (HR2 to HR5). Based on these functions, quantiles (annual precipitation values associated with a certain occurrence probability value) were determined, as well as precipitation deficit maps with 5, 10, 15, 20, 50 and 100 years return periods. The results indicate that meteorological drought events occur in the river basin with an average duration between 1.7 and 2.5 years, and with an average recurrence of 3.9 to 4.5 years; the longest drought period occurred between 1993-2002, and the period with the highest rainfall deficit was 2011-2012. The most affected regions are located in the states of Chihuahua and Coahuila, where the decrease levels in precipitation can exceed $80 \%$ of deficit, which indicates that for a return period of 100 years, more than half of the basin would show an extraordinary drought degree, which would have serious repercussions for the different socioeconomic sectors. 
Keywords: Meteorological drought, precipitation, regional frequency analysis, L-moments, Rio Grande River Basin.

Recibido 12/05/2020

Aceptado 22/09/2020

\section{Introducción}

De acuerdo con la Organización Meteorológica Mundial (OMM, 2006), la sequía se uede definir como un fenómeno climático recurrente que se caracteriza por ser un periodo de condiciones meteorológicas anormalmente secas, suficientemente prolongado como para que la falta de precipitaciones cause un grave desequilibrio hidrológico.

México es un país vulnerable a los efectos de las sequías. Por su ubicación geográfica, se encuentra en parte dentro del cinturón norte de los grandes desiertos del mundo, cuya existencia se debe fundamentalmente a la dinámica de la circulación general de la atmósfera y a particularidades que presenta la geomorfología del globo terrestre, que en conjunto producen los dos grandes cinturones de alta presión 
Tecnología y

Ciencias $\stackrel{\Xi}{\Im}$ Aua
2021, Instituto Mexicano de Tecnología del Agua

Open Access bajo la licencia CC BY-NC-SA 4.0

(https://creativecommons.org/licenses/by-nc-sa/4.0/)

atmosférica localizados en latitudes cercanas a los $30^{\circ}$ norte y sur (Ortega-Gaucin \& Velasco, 2013; Arreguín, López, Korenfeld, \& OrtegaGaucin, 2016a); debido a esto, gran parte de su territorio es árido o semiárido (52\%, con precipitaciones entre 300 y $600 \mathrm{~mm}$ ), por lo que percibe poca cantidad de lluvias, sobre todo en el norte del país; en contraste, el sureste es húmedo, con precipitaciones que superan en ocasiones los 2000 mm por año (Esparza, 2014; Conagua, 2016).

La cuenca del río Bravo es uno de los sistemas hidrológicos más importantes de México, donde el problema de la sequía es una situación recurrente (Linares, 2004; Ortega-Gaucin, 2013; Martínez, 2018). Sus condiciones hidrológicas se caracterizan por presentar un alto grado de variación en la disponibilidad del recurso hídrico debido a que la precipitación dentro de la cuenca es irregular y escasa, características que son influenciadas por la continentalidad, ubicación geográfica, complejidad orográfica y factores globales de circulación atmosférica (Brito-Castillo et al., 2010; Conagua, 2010; Núñez-López et al., 2013). Además, la variabilidad interanual de las precipitaciones en la región está vinculada de forma directa o indirecta con teleconexiones de variaciones de la temperatura superficial del mar en las regiones del Pacífico norte y del Atlántico. Es decir, fenómenos como El Niño Oscilación del Sur (ENSO) y la Oscilación Decadal del Pacífico (PDO) tienen gran influencia en las Iluvias estacionales (Rodríguez \& Pineda-Martínez, 2017).

La presión sobre los recursos hídricos en esta cuenca se ha incrementado en las últimas décadas debido a la creciente urbanización e industrialización de la zona fronteriza, que ha provocado una competencia por el agua entre los usuarios (en particular agrícolas) tanto en la parte 
alta y baja de la cuenca como en ambos lados de la frontera con EUA (Conagua, 2014). Por ser una cuenca binacional, el uso de las aguas del río Bravo y las de la mayoría de sus afluentes está sujeto a las regulaciones establecidas en el "Tratado sobre distribución de aguas internacionales entre los Estados Unidos Mexicanos y los Estados Unidos de América de 1944". Por ello, debido a la obligación de satisfacer tanto las demandas de la población como de cumplir con el Tratado mencionado, aunado a la creciente información sobre los efectos del cambio climático en los patrones y cantidad de lluvia y escurrimiento en esa región (Arreguín, López, Velázquez, \& López, 2013; Martínez, 2018), los diferentes sectores socioeconómicos son altamente vulnerables a los impactos producidos por las sequías y el uso inapropiado del agua.

En la literatura se reconoce que existen diferentes tipos de sequías dependiendo de su origen e impactos que generan. Entonces se considera tratar la sequía desde distintos enfoques como el meteorológico, el agrícola, el hidrológico y el socioeconómico (Wilhite \& Glantz, 1985; Valiente, 2001; OMM, 2006). La presente investigación está centrada en el análisis específico de la sequía meteorológica, es decir, el déficit de precipitaciones con respecto a los niveles considerados como normales dentro del área de estudio. Cuando esta anomalía se prolonga en el tiempo y se combina con las presiones antrópicas de demanda de aguas superficiales y subterráneas provoca serios impactos en los distintos sectores socioeconómicos y ambientales.

Debido a los efectos negativos de esta amenaza climática, en los últimos años se ha ido generando un cambio de enfoque en su tratamiento, que va de la atención reactiva de los impactos causados por 
la sequía a la adopción de medidas adecuadas para prevenir y mitigar sus efectos. Este nuevo enfoque se ha dado, en especial, en el contexto de las estrategias de gestión y reducción del riesgo de desastres en la planificación del desarrollo (Bass, Ramasamy, Dey-Deprick, \& Batista, 2008). En este contexto, el riesgo de un desastre por eventos de sequía ha sido definido como el producto del peligro o amenaza (representada por la probabilidad de ocurrencia de la sequía), y el grado de vulnerabilidad del sistema afectado (Wilhite, 2000; Ortega-Gaucin, De-laCruz, \& Castellano, 2018).

Independientemente del enfoque utilizado para determinar el riesgo de los sistemas sociales y ambientales a los eventos de sequía, el común denominador en todas las metodologías lo constituye la necesidad de estimar la probabilidad de ocurrencia de un evento de sequía de determinada magnitud (Carrao, Naumann, \& Barbosa, 2016). Por lo general, las características más comunes de la sequía como la intensidad, duración y distribución geográfica se obtienen mediante el uso de índices e indicadores de diversa resolución espacial y temporal (OMM-GWP, 2016). Pero la probabilidad de ocurrencia de la sequía puede ser obtenida mediante las herramientas de la hidrología probabilística. No obstante, las características propias de las regiones áridas y semiáridas, con gran variabilidad espacial y temporal de las precipitaciones, influencia de factores externos de variabilidad decadal, como los efectos del ENSO, y la reducida disponibilidad de registros, imponen ciertas restricciones a la elección del método más adecuado para el ajuste probabilístico (Ka Ima \& Franks, 2003). 
Tecnología y

Ciencias $₫$ Agua
2021, Instituto Mexicano de Tecnología del Agua

Open Access bajo la licencia CC BY-NC-SA 4.0

(https://creativecommons.org/licenses/by-nc-sa/4.0/)

Así, para resolver los problemas relacionados con la limitada y asimétrica disponibilidad de datos en el espacio y en el tiempo, o la ausencia de registros de una longitud adecuada que permita realizar estimaciones de frecuencia con cierto grado de confiabilidad, surge la necesidad de estudiar, validar y difundir metodologías alternativas, de fácil implementación práctica, que permitan llevar a cabo mejores estimaciones de la frecuencia con la cual se espera que ocurran las sequías, y que generen información más confiable para la toma de decisiones (UNESCO, 2010). En este tenor, el análisis regional de frecuencia de sequías basado en el método de L-momentos ha sido considerado como una de las mejores alternativas para la evaluación, estimación y mapeo de la probabilidad de ocurrencia de eventos de sequía en regiones áridas y semiáridas con limitada disponibilidad de registros de lluvia debido a que compensa la carencia de información en el tiempo, por su abundancia en el espacio (Hosking \& Wallis, 1997).

Por tanto, en los últimos años el método basado en L-momentos ha sido utilizado para determinar las características de la sequía meteorológica en distintas partes del mundo (Abolverdi \& Khalil, 2010; UNESCO, 2010; Acuña, Felipe, Ordoñez, \& Arboleda, 2011; Acuña, Felipe, \& Fernández, 2015; Naranjo, 2011; Eslamian, Hassanzadeh, AbediKoupai, \& Gheysari, 2012; Paredes, La-Cruz, \& Guevara, 2014; La-Cruz, 2015); pero en México sólo ha sido aplicado con este propósito en la región noroeste del país (Hallack \& Ramírez, 2010; De-la-Cruz \& OrtegaGaucin, 2019). Sin embargo, este método también se aplica a la solución de problemas que requieren la estimación de frecuencia de ocurrencia de una serie de fenómenos, como: precipitaciones máximas diarias y 
horarias, caudales máximos y mínimos, velocidad del viento, radiación solar, etcétera. En el ámbito internacional este método ha sido empleado para analizar los eventos de crecidas y precipitaciones máximas (Schaefer, Barker, Taylor, \& Wallis, 2007; Norbiato, Borga, Sangati, \& Zanon, 2007; Báez, Prieto, \& Aroche, 2016), al igual que para temperaturas máximas (Núñez-Galeano \& Giraldo-Osorio, 2016), y caudales de ríos (Vich, Norte, \& Lauro, 2014; Campos-Aranda, 2014; Castillo \& Ortiz, 2015).

En tal contexto, la presente investigación tiene como objetivo presentar los fundamentos y la aplicación del método basado en Lmomentos para analizar el comportamiento de las sequías meteorológicas, así como determinar las características de los periodos secos y crear mapas de déficit de precipitación con diferentes periodos de retorno, para lo cual se utiliza como caso de estudio la cuenca del río Bravo, México. Con ello se pretende contribuir a la comprensión del fenómeno de la sequía en esta región estratégica para el desarrollo del país, y dar respuesta a algunas interrogantes como las siguientes: ¿Cada cuántos años se puede esperar que un evento de sequía severa afecte el territorio de la cuenca?, ¿existen zonas más propensas que otras al acontecimiento de una sequía extrema o excepcional?, ¿cuál ha sido el periodo más seco de las últimas décadas?, y ¿cuál es la probabilidad de que un año cualquiera pueda ocurrir un déficit de más del $80 \%$ de la precipitación anual en diferentes zonas de la cuenca?

Lo cierto es que aunque en la práctica puede ser sumamente difícil predecir la ocurrencia de un periodo de sequía, el conocimiento de la frecuencia, duración e intensidad de las sequías históricas registradas, así 
como su probabilidad de ocurrencia, pueden ayudar a los planeadores y operadores de los sistemas hidráulicos e hidrológicos a anticiparse a los efectos de la sequía (Wilhite, 2000), y servir de base para la adecuada planeación y toma de decisiones sobre el manejo de los recursos hídricos en la cuenca.

\section{Materiales y métodos}

\section{Descripción del área de estudio}

La cuenca del río Bravo tiene la característica de ser binacional, ya que está ubicada en la frontera entre México y EUA (Figura 1). Pertenece a la Región Hidrológica No. 24 Bravo-Conchos, y tiene una extensión territorial de $226275 \mathrm{~km}^{2}$, lo que corresponde a un $11 \%$ del total del territorio nacional. Geográficamente se ubica entre los $31^{\circ} 50^{\prime}$ y $24^{\circ} 55^{\prime}$ de latitud norte y los $107^{\circ} 45^{\prime}$ y $97^{\circ} 15^{\prime}$ de longitud oeste, abarcando parte del estado de Chihuahua, una porción de los estados de Durango y Tamaulipas, y gran parte de los estados de Coahuila y Nuevo León (Semarnat, 2011). 


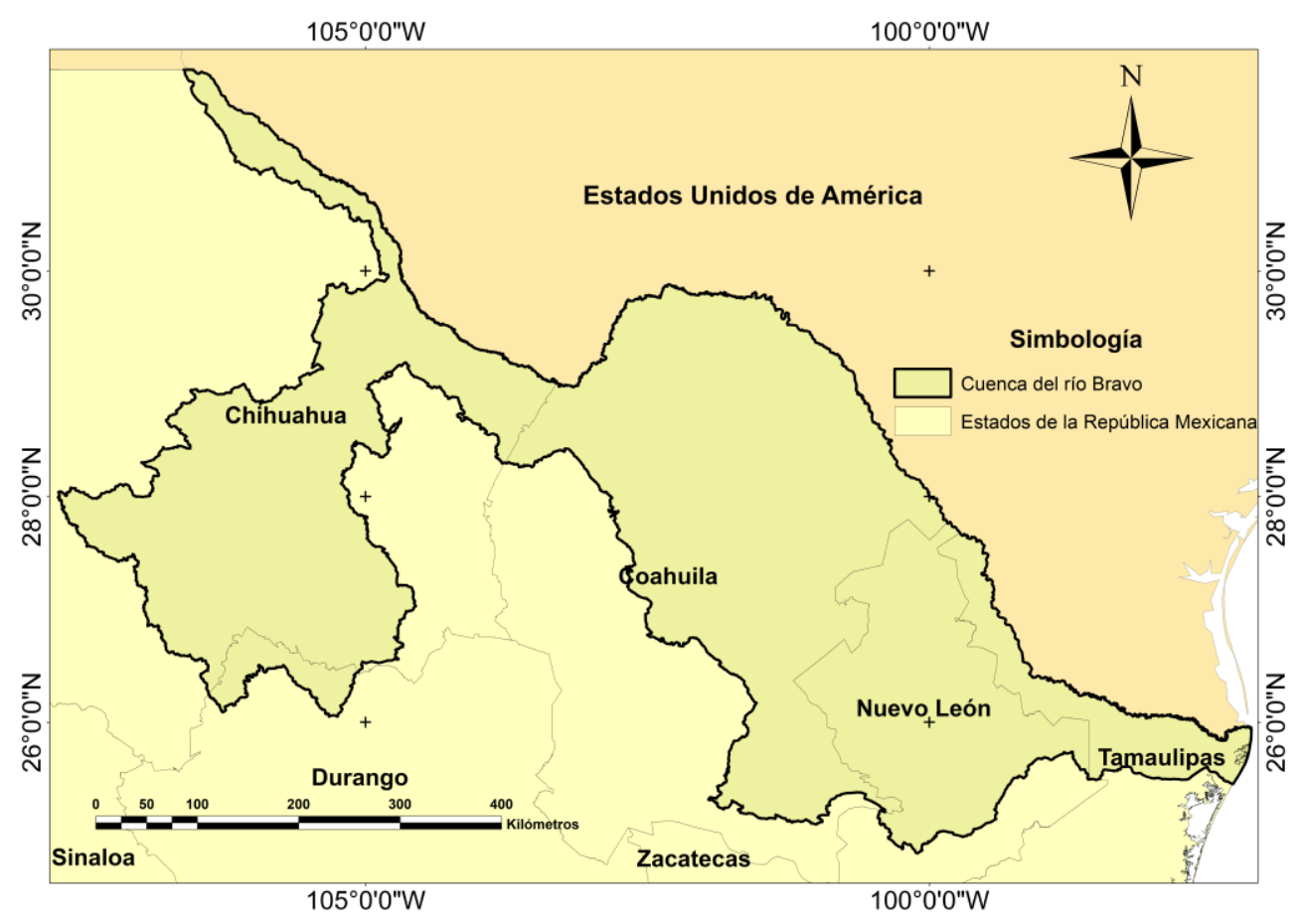

Figura 1. Ubicación de la cuenca del río Bravo, sección mexicana.

Esta cuenca se divide en cuatro subregiones hidrológicas: seis tributarios, alto, medio y bajo Bravo; y se integra a su vez por 37 subcuencas (Semarnat, 2011); registra una precipitación media anual (PMA) de 371.7 mm, lo que corresponde al $50 \%$ de la PMA del país (739.8 $\mathrm{mm}$ ), de acuerdo con el periodo 1981-2010 (Conagua, 2018). Sin embargo, ese valor de precipitación es muy variable tanto temporal como geográficamente. En específico se ha documentado que los periodos fríos (cálidos) de ENSO producen un déficit (aumento) invernal de la lluvia en la mayor parte de México y el suroeste de EUA (Seager et al., 2009); y la 
disminución de la precipitación se asocia con un debilitamiento de los flujos de humedad durante el verano en fases negativas de PDO, aunque mayormente en invierno (Magaña, Vázquez, Pérez, \& Pérez, 2003). Las Iluvias que producen escurrimientos importantes en esta cuenca son producto de la influencia de ciclones tropicales, provenientes sobre todo del Golfo de México (Brito-Castillo et al., 2010; Núñez-López et al., 2013). Por ello, con una disponibilidad limitada de agua, el desarrollo de la región, una de las más dinámicas del país, ha propiciado la sobreexplotación de los acuíferos y la presencia de conflictos entre usuarios por este recurso natural.

\section{Selección de estaciones climatológicas}

Para la obtención de los registros mensuales históricos de precipitación se seleccionaron estaciones de la base de datos climatológica nacional (sistema CLICOM), perteneciente al Servicio Meteorológico Nacional (SMN). El criterio de selección utilizado fue el recomendado por la OMM, de elegir estaciones que cuenten con 80 \% o más de datos disponibles dentro del periodo de análisis (1984-2013). Así, de las 147 estaciones climatológicas funcionales al momento del estudio, se eligieron 90 que cumplieron el criterio mencionado (ver Anexo 1). En la Figura 2 se muestra su distribución espacial, donde se puede observar que aunque se 
encuentran en cierto grado distribuidas dentro de la cuenca hay una ausencia significativa de ellas en la parte central y en los extremos de la misma. No obstante, mediante los procedimientos de análisis que se describen en los apartados siguientes, es posible subsanar esta carencia de información.

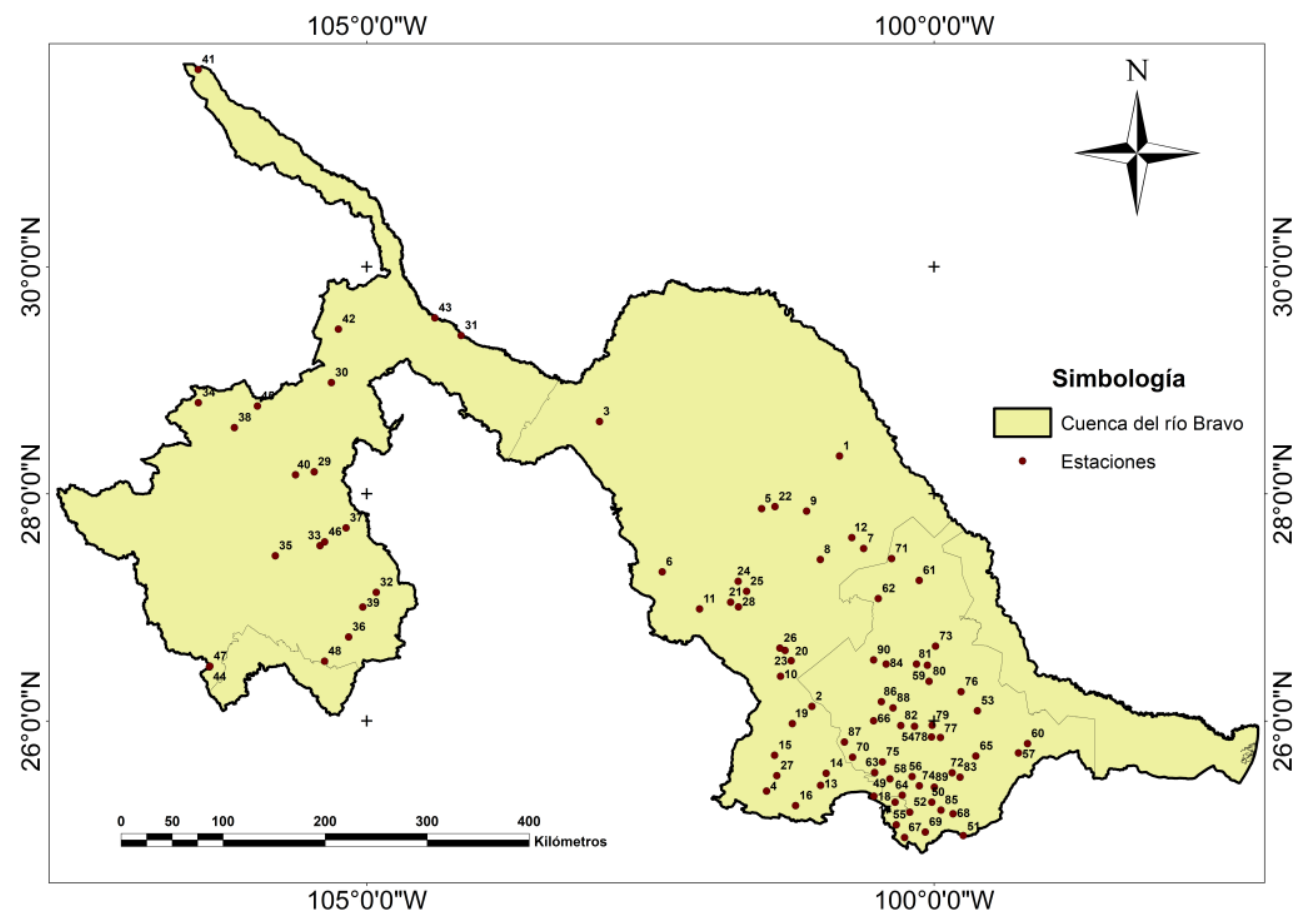

Figura 2. Ubicación de las estaciones climatológicas seleccionadas.

\section{Fundamentos del análisis regional de frecuencias basado en L-momentos (ARF-LM)}


Los L-momentos $\left(\lambda_{i}\right)$ son combinaciones lineales de los momentos de probabilidad ponderada $\left(\beta_{i}\right)$, desarrollados por Greenwood, Landwehr, Matalas y Wallis (1979), y éstos son parámetros estadísticos asociados con los datos ordenados. El empleo de los L-momentos supone el uso de un sistema alternativo a los métodos tradicionales al momento de describir las formas de las funciones de distribución. Surgen por combinaciones lineales de momentos ponderados probabilísticamente. Las ecuaciones para su cálculo son (Stedinger, Vogel, \& FoufoulaGeorgiou, 1993; Hosking \& Wallis, 1997):

$\lambda_{1}=\beta_{o}$

$\lambda_{2}=2 \beta_{1}-\beta_{o}$

$\lambda_{3}=6 \beta_{2}-6 \beta_{1}+\beta_{o}$

$\lambda_{4}=20 \beta_{3}-30 \beta_{2}+12 \beta_{1}-\beta_{o}$

El momento lineal de primer orden $\left(\lambda_{1}\right)$ es la media de la variable; el segundo momento $\left(\lambda_{2}\right)$ se refiere a la escala de distribución en el que se indica el grado de dispersión de los datos; el momento lineal de tercer 
orden $\left(\lambda_{3}\right)$ representa la asimetría; por último, el cuarto momento lineal $\left(\lambda_{4}\right)$ se refiere al apuntamiento (también llamado curtosis) que mide cuán escarpada o achatada está la distribución de los datos.

La comparación de los registros de precipitación de las estaciones climatológicas se realiza por contraste de los L-momentos de manera adimensional, calculando los L-ratios $\left(\tau_{1}\right)$. Estos se obtienen dividiendo aquellos momentos lineales de mayor escala, $\lambda_{3}$ y $\lambda_{4}$, por la escala de distribución $\lambda_{2}$. De esta manera se logra obtener una forma de la distribución independiente a su escala de medida, lo que permite evaluar de modo equitativo estaciones con diferentes registros de series. Los valores más importantes son los siguientes:

$L-C V: \tau_{2}=\lambda_{2} / \lambda_{1}$

$L-$ Asimetría $: \tau_{3}=\lambda_{3} / \lambda_{2}$

L-Custosis: $\tau_{4}=\lambda_{4} / \lambda_{2}$

El ARF-LM asume que un conjunto de estaciones pluviométricas dentro de un área determinada forman una región homogénea si y sólo si sus distribuciones de frecuencias son idénticas, variando únicamente por un factor de escala específico en cada estación, lo que permite agregar todas las estaciones que forman parte de una región homogénea, con el objeto de mejorar la precisión en las estimaciones de la función de 
Tecnología y

\section{Ciencias Agua}

2021, Instituto Mexicano de Tecnología del Agua

Open Access bajo la licencia CC BY-NC-SA 4.0

(https://creativecommons.org/licenses/by-nc-sa/4.0/)

probabilidad cuantílica en dichas estaciones (Hosking \& Wallis, 1997; Wallis, Schaefer, Barker, \& Taylor, 2007).

El ARF-LM utiliza modelos de distribución de tres o más parámetros, por lo tanto, es más robusto que los modelos de distribución empleados en la hidrología probabilística clásica, los cuales suelen ser de uno o dos parámetros.

La metodología ARF-LM -adaptada de la UNESCO (2010) para realizar esta investigación- considera la aplicación de cinco etapas (Figura 3): 1) revisión y control de calidad de los datos; 2 ) identificación de regiones homogéneas; 3 ) selección de la distribución de frecuencias; 4) estimación de cuantiles, y 5) mapeo. La primera etapa se realizó con ayuda de la paquetería Climatol, ejecutada en el lenguaje de programación $R$; para las etapas 2 a 4 se utilizó el programa L-RAP ( $L$ Moment Regional Analysis Program); y en la etapa 5 se usó el software ArcGis ${ }^{\circledR}$, tal como se describe en los incisos subsecuentes.

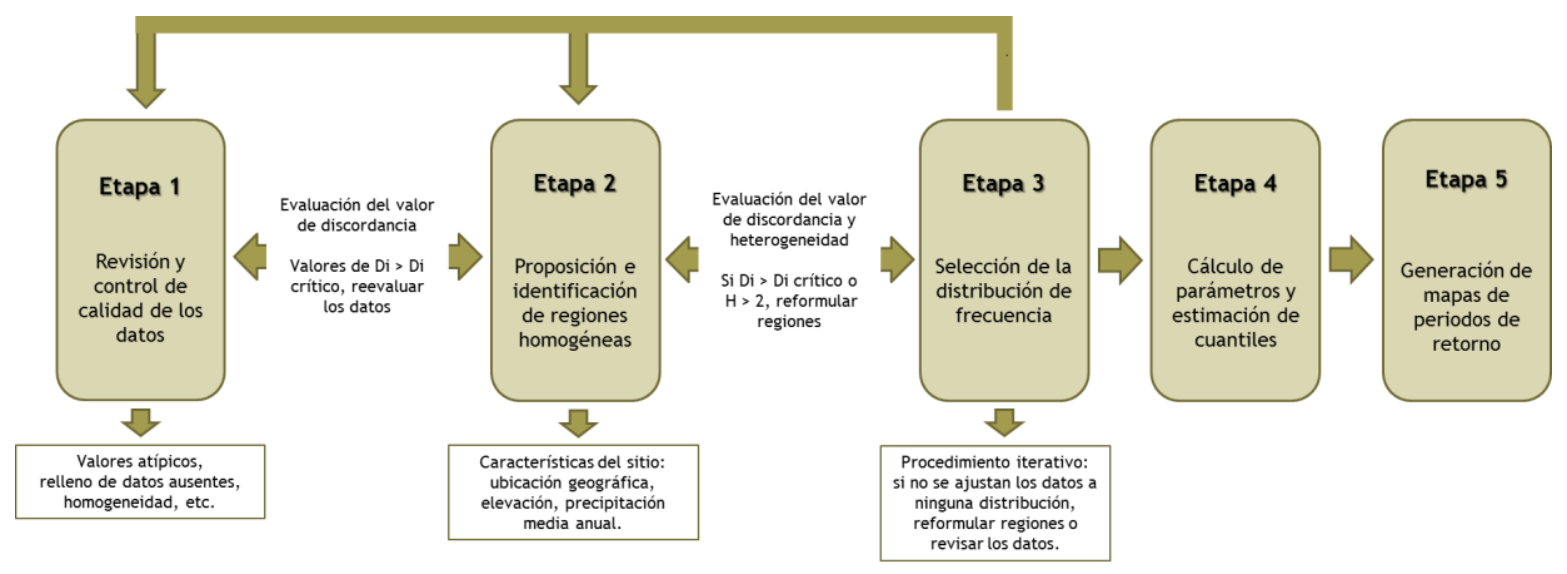


Figura 3. Diagrama de flujo de las etapas del ARF-LM. Adaptada de la UNESCO (2010).

1. Revisión y control de calidad de los datos: el objetivo de esta etapa es eliminar los registros dudosos asociados con errores de registro y/o transcripción, así como rellenar datos faltantes. En este caso, el proceso de control de calidad, homogeneización y relleno de datos ausentes de las series de precipitación se realizó mediante el software Climatol en su versión 3.1.1 (Guijarro, 2018), ejecutado en lenguaje $R$. Esta herramienta permite rellenar huecos de las series climáticas mediante el método de Paulhus y Kohler (1952), por medio de una serie de procesos iterativos que normalizan los datos mediante la división por sus valores medios $(x=X / \mu x)$, además de restar las medias $(x=X-\mu x)$, o mediante una estandarización completa $[x=(X-\mu x) / \sigma x]$, denominando $\mu x$ y $\sigma x$ a la media y desviación típica de una serie $X$, respectivamente. Asimismo, permite detectar valores anómalos, que puedan deberse a errores de captura de la información a través de la prueba de homogeneidad denominada Standard Normal Homogeneity Test (Alexandersson, 1986). Para aplicar los procesos mencionados en la cuenca bajo estudio, ésta se dividió en dos grandes zonas (Figura 4) con la finalidad de lograr un mejor ajuste de las series a través de dos procesos independientes, siguiendo las recomendaciones de Guijarro (2018) acerca de la cercanía o contigüidad de las estaciones. En la zona A (color rojo) se ubican 20 estaciones climatológicas y en la zona B (color azul) se localizan 70 estaciones. Estos procesos dieron como resultado 
Ciencias $₫$ Agua
2021, Instituto Mexicano de Tecnología del Agua

Open Access bajo la licencia CCBY-NC-SA 4.0

(https://creativecommons.org/licenses/by-nc-sa/4.0/)

nuevas series reconstruidas que después se analizaron y aceptaron para su uso en el ARF-LM por medio del software $L-R A P$.

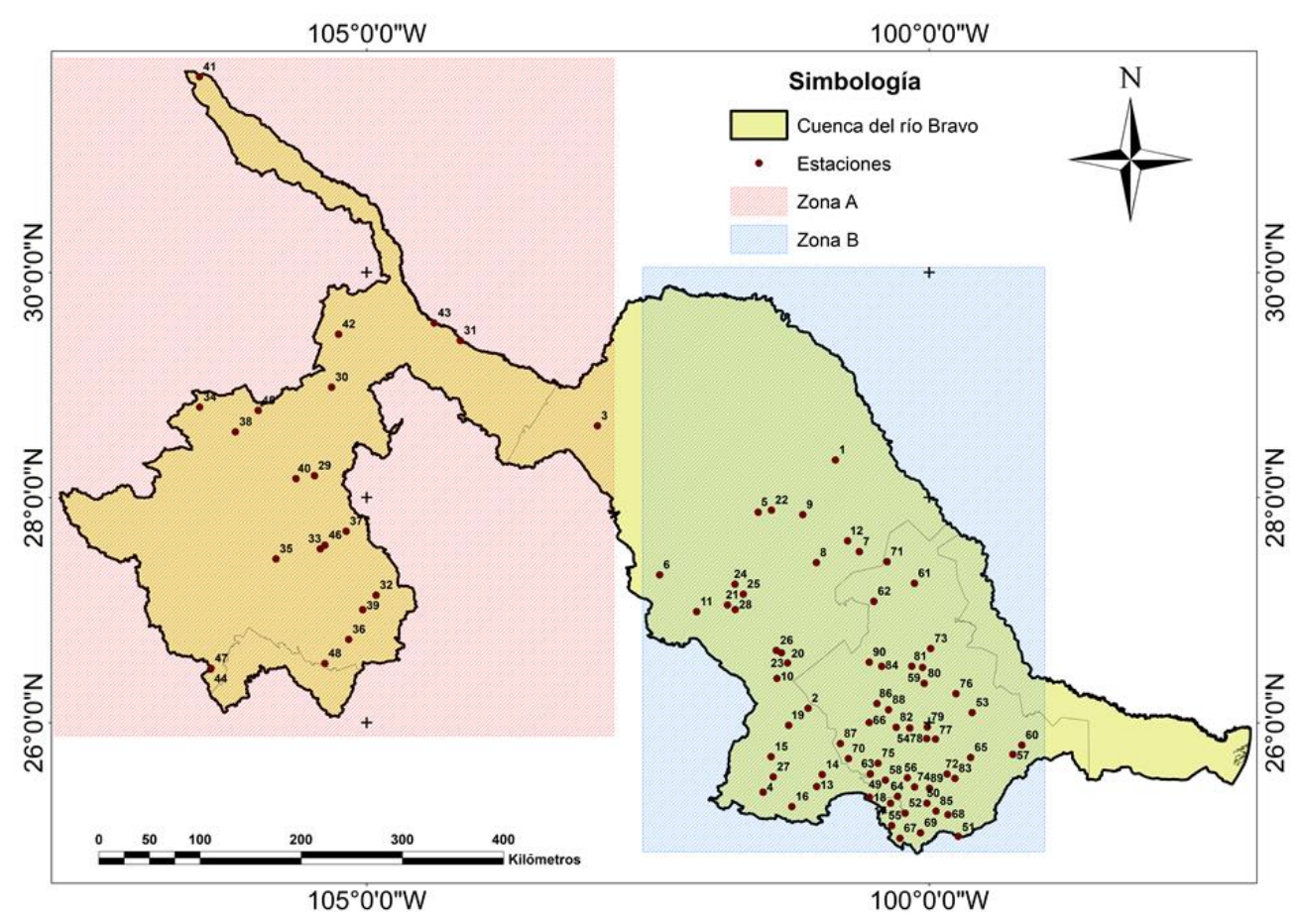

Figura 4. División de la cuenca para la revisión y el control de calidad de los datos de precipitación mediante Climatol.

2. Identificación de regiones homogéneas $(R H)$ : el objetivo de esta etapa es formar regiones que satisfagan la condición de homogeneidad. Se definiría a esta región como aquella conformada por un conjunto de estaciones climatológicas donde se puede asumir que la función de distribución de probabilidades que rige la precipitación es la misma para 
cada una de ellas. La regionalización es un proceso iterativo que consiste de dos pasos:

a) El primer paso es determinar regiones a priori, identificando estaciones en un espacio geográfico con características climatológicas y topográficas similares, y utilizando la magnitud de la PMA como criterio de agrupamiento de las estaciones. Este criterio ha sido recomendado como el más adecuado para agrupar estaciones con miras a un análisis regional de frecuencia de precipitaciones en el contexto de la caracterización probabilística de eventos de sequía (UNESCO, 2010). Así, dado un conjunto de estaciones en una región, es necesario verificar que las estaciones que la integran no presenten propiedades que las hagan discordantes con el resto. Para ello, Hosking y Wallis (1997) presentan una medida de discordancia, la cual permite evaluar el grado en que los L-momentos de una estación se adaptan de forma significativa al patrón medio de los L-momentos regionales:

$D_{i}=\frac{1}{3} N(\mathrm{ui}-\overline{\mathrm{u}})^{T} A^{-1}(\mathrm{ui}-\overline{\mathrm{u}})$

donde $D_{i}$ define la media de las discordancias de cada estación $i$. Suponiéndose que la región presenta $N$ estaciones, donde $u i=\left[t^{(i)} t_{3}{ }^{(i)} t_{4}{ }^{(i)}\right]^{T}$ son $N$ vectores columna, que contiene los valores de $t_{1} t_{3}$ y $t_{4}$ de los Lmomentos de cada estación $i$, donde el superíndice $T$ indica la transposición del vector. Siendo $\bar{u}$ el vector promedio de la región 
Tecnología y

Ciencias $₫$ Agua
2021, Instituto Mexicano de Tecnología del Agua

Open Access bajo la licencia CC BY-NC-SA 4.0

(https://creativecommons.org/licenses/by-nc-sa/4.0/)

homogénea analizada y $A$ la matriz de sumas de cuadrados y productos cuadrados de las estaciones $\left[\left(\mathrm{u}_{\mathrm{i}}-\overline{\mathrm{u}}\right)\left(\mathrm{u}_{\mathrm{i}}-\overline{\mathrm{u}}\right)^{T}\right]$ de la misma RH.

Para evaluar si una estación es discordante o no en función de un determinado número de estaciones, se establecen los valores de referencia de $D_{i}$ que se presentan en la Tabla 1.

Tabla 1. Valores críticos de discordancia según Hosking y Wallis (1997).

\begin{tabular}{|c|c|c|c|}
\hline $\begin{array}{c}\text { Núm. de } \\
\text { estaciones por } \\
\text { región }\end{array}$ & $\begin{array}{c}\text { Valor } \\
\text { crítico }\end{array}$ & $\begin{array}{c}\text { Núm. de } \\
\text { estaciones por } \\
\text { región }\end{array}$ & $\begin{array}{c}\text { Valor } \\
\text { crítico }\end{array}$ \\
\hline 5 & 1.333 & 11 & 2.632 \\
\hline 6 & 1.648 & 12 & 2.757 \\
\hline 7 & 1.917 & 13 & 2.869 \\
\hline 8 & 2.140 & 14 & 3.000 \\
\hline 9 & 2.329 & $>15$ & \\
\hline 10 & 2.491 & & \\
\hline
\end{tabular}

b) El segundo paso es realizarlas pruebas de heterogeneidad, en donde se detecta si las estaciones dentro de la región formada en el paso anterior pueden ser caracterizadas como homogéneas. La medida de heterogeneidad para la aceptación o rechazo de las regiones homogéneas propuestas es el estadístico $H$ (Hosking \& Wallis, 1997), que mide la 
variabilidad relativa del coeficiente de L-Variación ( $L-C V$, en inglés), observado a partir de la muestra, el cual se determina mediante:

$H=\frac{\left(V-\mu_{v}\right)}{\sigma_{v}}$

donde $V$ es la desviación estándar de los $L-C V$ locales, $\mu_{v}$ representa la media de $V$, y $\sigma_{v}$ es la desviación estándar de $V$.

De acuerdo con Wallis et al. (2007), una región puede ser considerada como homogénea, si los valores estadísticos de la prueba son menores a 2.0 (Tabla 2).

Tabla 2. Valores críticos de heterogeneidad según Wallis et al. (2007).

\begin{tabular}{|l|c|}
\hline \multicolumn{1}{|c|}{ Heterogeneidad (H) } & Valores de $\boldsymbol{H}$ \\
\hline Homogénea & $H<2$ \\
\hline Posiblemente heterogénea & $2<H<3$ \\
\hline Heterogénea & $H>3$ \\
\hline
\end{tabular}

En caso de que la prueba de heterogeneidad falle, se revisan las medidas de discordancia para cada estación y se formula una nueva región reagrupando las estaciones discordantes. El proceso iterativo culmina cuando la región puede ser clasificada como homogénea. 
3. Selección de la distribución de frecuencias: una vez que una región ha aprobado la condición de homogeneidad es posible determinar la distribución de probabilidad de mejor ajuste con base tanto en el uso del diagrama de los L-momentos-ratios regionales como en la prueba de bondad de ajuste $Z^{\text {DIST }}$ (Ecuación (10)), así como estimar los parámetros de ubicación, escala y forma de esta distribución.

$Z^{D I S T}=\frac{\left(t_{4}^{D I S T}-t_{4}^{R}-B_{4}\right)}{\sigma_{4}}$

donde $t_{4}{ }^{R}$ representa el valor medio regional de L-Curtosis; $B_{4}$ es el sesgo de $t_{4}{ }^{R} ; \sigma_{4}$ es la desviación estándar de $t_{4}{ }^{R} ;$ y $t_{4}{ }^{D I S T}$ es el coeficiente de LCurtosis de la distribución ajustada, en el que DIST hace referencia a las funciones de distribución de probabilidades (FDP) sugeridas por Hosking y Wallis (1997) que son: Logística Generalizada (GL), General de Valores Extremos (GEV), Generalizada Normal (GN), Kappa de cuatro parámetros (Gaucho), LogPearson Tipo III (LP3) y Generalizada de Pareto (GP).

El criterio para declarar una distribución como satisfactoria es si el valor absoluto de $Z^{D I S T}$ es menor o igual que 1.64, es decir, $Z^{D I S T} \leq|1.64|$ (Hosking \& Wallis, 1997).

4. Estimación de cuantiles: el objetivo de esta etapa es determinar los cuantiles, es decir, los valores de la precipitación anual asociados con un determinado valor de probabilidad de ocurrencia. Seleccionadas las regiones y las distribuciones de frecuencia más apropiadas para cada caso, se procede a determinar los parámetros necesarios para el cálculo 
de los cuantiles o la función de cuantiles completa para un sitio específico. Esto es:

$Q_{i}(F)=\mu_{i} q(F) i=1,2 \ldots n$

Es decir, la función de cuantiles del sitio de interés $Q_{i}(F)$ se determina a partir de $q(F)$, que es la función de cuantiles regional adimensional (curva de crecimiento regional) estimada mediante el análisis regional de frecuencias y multiplicada por un factor de escala $\left(\mu_{i}\right)$, que puede ser el promedio de la variable analizada para el sitio de interés (UNESCO, 2010), siendo $i$ cada una de las estaciones y $n$ el número total de estaciones de la región. Mediante esta curva de crecimiento regional se muestra la relación entre la precipitación media local/precipitación media regional y la probabilidad de no excedencia anual, a partir de la cual se puede estimar la probabilidad de no excedencia o el periodo de retorno ( $\operatorname{Tr}$ ) de cualquier evento de interés a escala anual (La-Cruz, 2015).

5. Mapeo: una de las ventajas que presenta el método de ARF-LM es la facilidad de implementar el mapeo espacial de los cuantiles, probabilidades o periodos de retorno, considerando aquellos sitios medidos y no medidos. Los mapas obtenidos son de gran valor para la toma de decisiones (Wallis et al., 2007; Schaefer et al., 2007). En este caso, se generaron mapas de sequía meteorológica asociados con diferentes periodos de retorno (5, 10, 15, 20, 50 y 100 años) mediante el uso del sistema de información geográfica $\operatorname{ArcGIS} \otimes$. El método de 
interpolación utilizado en todos los mapas fue el de "kriging", el cual se basa en los fundamentos de la teoría geoestadística o de variables georreferenciadas, y no parte del supuesto de normalidad de la variable analizada (Oliver \& Webster, 1990). Esto representa una ventaja en el manejo de todo tipo de variables que no se distribuyen de manera normal, ni en el espacio, ni en el tiempo, como es el caso de la precipitación pluvial.

\section{Resultados y discusión}

\section{Revisión y control de calidad de los datos}

De acuerdo con el análisis realizado de los datos mensuales de precipitación mediante la paquetería Climatol, la disponibilidad de datos en el periodo 1984-2013 fue de $92 \%$ en promedio para las estaciones de la zona $A$, y de $88 \%$ en promedio para las de la zona $B$, tal como se muestra en la Figura 5, donde se observa que las estaciones de la zona B presentan mayor cantidad de huecos (espacios blancos) dentro del periodo de registro. 
2021, Instituto Mexicano de Tecnología del Agua

Tecnología y

Ciencias $₫$ Agua
Open Access bajo la licencia CC BY-NC-SA 4.0

(https://creativecommons.org/licenses/by-nc-sa/4.0/)
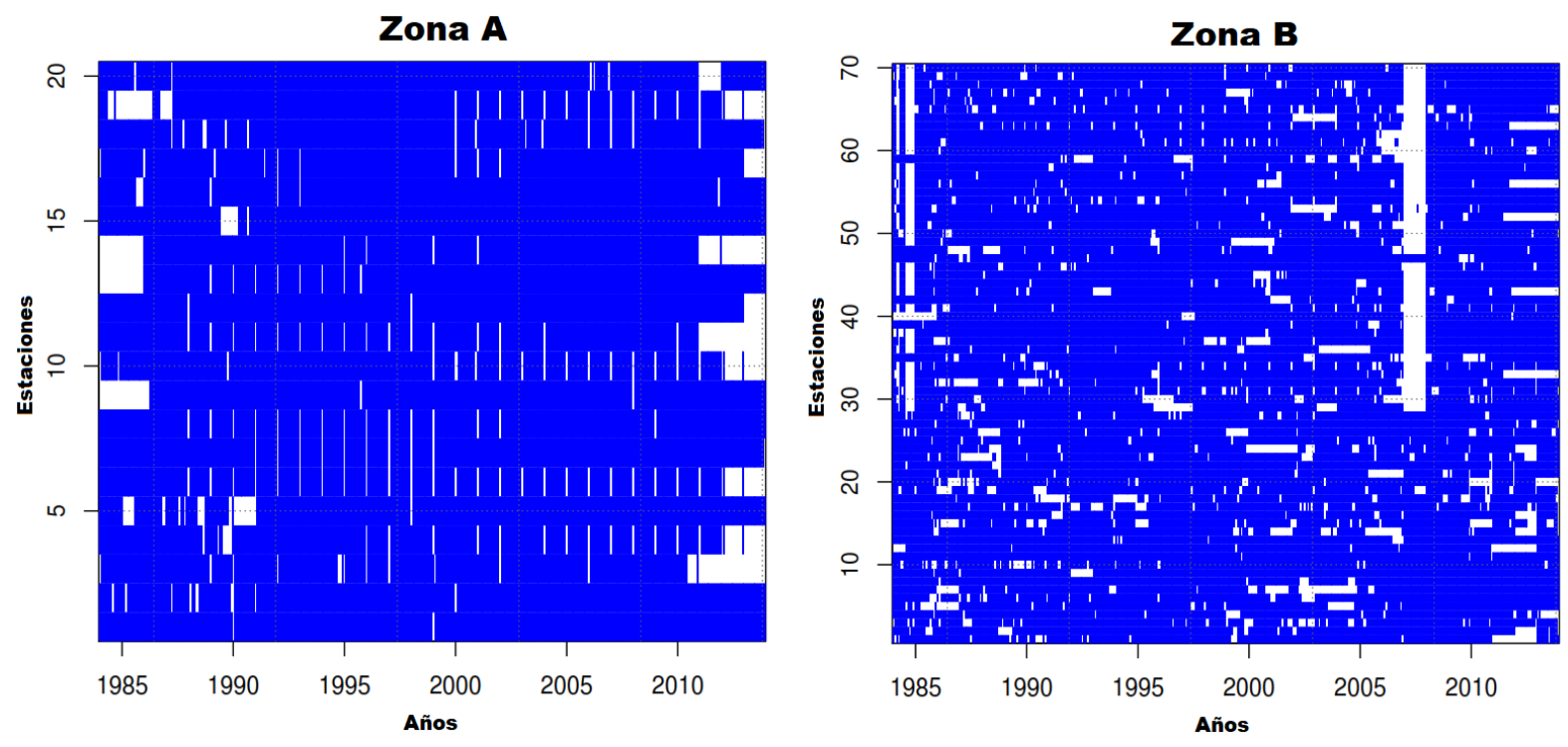

Figura 5. Disponibilidad de datos mensuales de precipitación por estaciones climatológicas (1984-2013).

Después de llevar a cabo el proceso de homogeneización y relleno de datos faltantes mediante la herramienta mencionada, se obtuvieron las series reconstruidas para cada estación, generadas a partir de los datos originales. A manera de ejemplo, en la Figura 6 se muestra la gráfica correspondiente a la estación climatológica "La Rosa", en la que se aprecian las medias anuales móviles de las series reconstruidas (parte superior), con los datos originales en color negro y los rellenados en diferentes colores (rojo, verde, azul cielo y azul marino) para cada serie resultante del proceso iterativo. La parte inferior muestra las correcciones aplicadas a las series trazadas en los mismos colores. De esta manera, 
Tecnología y

Ciencias $₫$ Agua
2021, Instituto Mexicano de Tecnología del Agua

Open Access bajo la licencia CC BY-NC-SA 4.0

(https://creativecommons.org/licenses/by-nc-sa/4.0/)

en todos los casos se seleccionaron y utilizaron aquellas series que representan de mejor manera los valores originales que fueron ingresados a la paquetería Climatol que, para el caso del ejemplo en cuestión, corresponden a la serie en color rojo, cuyo factor de corrección es el más bajo.

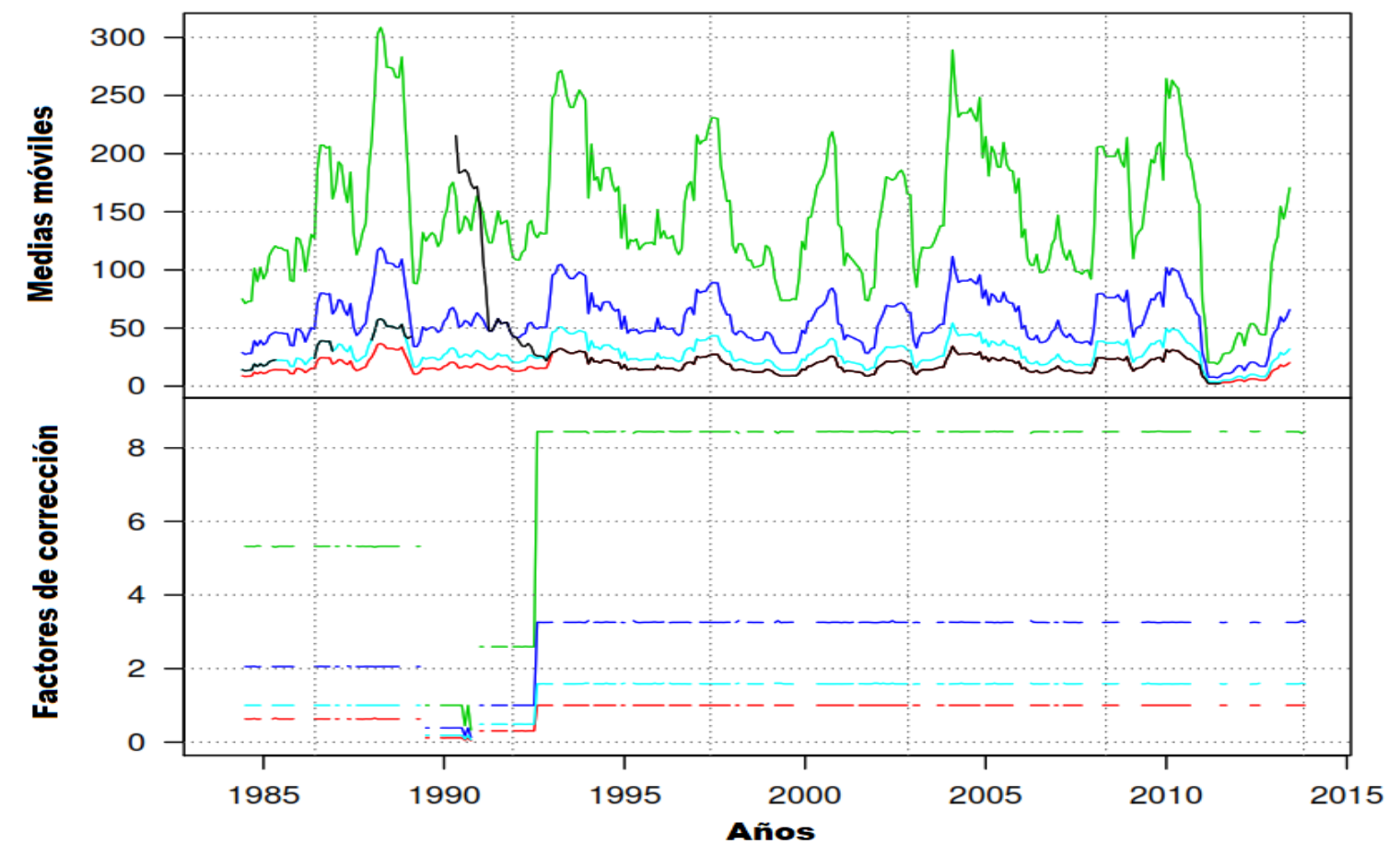

Figura 6. Reconstrucción de series completas de datos de precipitación para una estación climatológica mediante Climatol.

\section{Distribución espacio-temporal de la precipitación}


Como resultado del procesamiento de la información obtenida en la etapa anterior, en la Figura 7 se presentan los mapas que ilustran la distribución de la PMA dentro de la cuenca del río Bravo para el periodo de estudio, generados a partir de las series de precipitación originales y reconstruidas, donde se observa que las isoyetas tienen ciertos cambios (aunque menores) de un mapa a otro. En ambos se aprecia cómo varía la Iluvia en las distintas regiones de la cuenca, presentando los valores más bajos hacia el noroeste (150-250 mm), en las colindancias de Chihuahua con EUA, y en la porción central (251-450 mm), abarcando la mayor parte del estado de Coahuila. Y los más altos en el sur y sureste, en la parte alta de la subcuenca del río Conchos (451-650 mm) y hacia los estados de Nuevo León y Tamaulipas (651-1 050 mm). Este patrón de distribución espacial de la precipitación concuerda con lo señalado por Brito-Castillo et al. (2010), quienes afirman que en esa región del monzón en particular existe un patrón de distribución de la lluvia de sur a norte y de este a oeste. Además, de acuerdo con Núñez-López et al. (2013), esta distribución pone en evidencia la relación que guardan los rasgos geográficos, la distancia continental a las zonas marítimas y la complejidad del relieve con la precipitación que ocurre sobre la cuenca del río Bravo. 

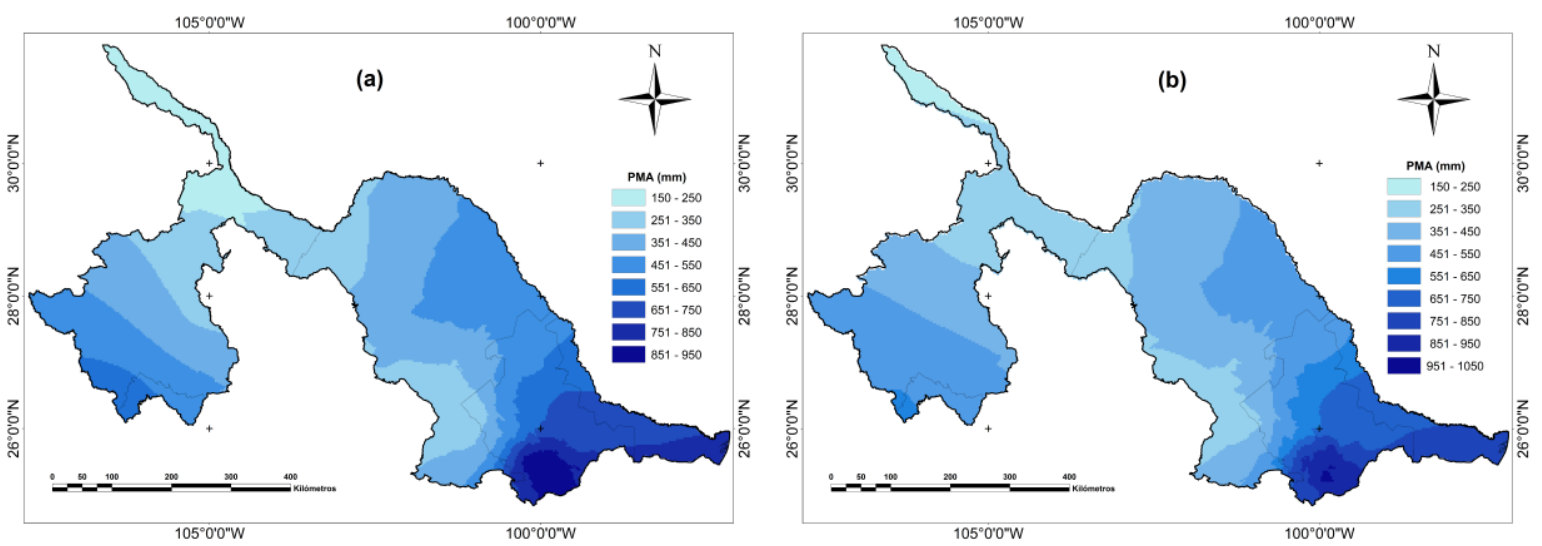

Figura 7. Mapas de precipitación media anual en la cuenca del río Bravo generados a partir de las series originales (a) y reconstruidas (b).

De igual forma, en la Figura 8 se presenta la distribución temporal de la lluvia a lo largo del año, donde se aprecia que el régimen de precipitación se caracteriza por la presencia de un periodo de lluvias abundantes claramente definido en el verano, entre los meses de junio a septiembre, siendo septiembre el mes más húmedo con $97.4 \mathrm{~mm}$ en promedio. La precipitación media acumulada durante los cuatro meses Iluviosos representa aproximadamente el $62 \%$ de la precipitación total anual captada en la cuenca. Estos datos son similares a los reportados por Conagua (2018), la cual indica que el $61.4 \%$ de la lluvia ocurre en esos meses del año, y septiembre es el más lluvioso de todos. 
Tecnología y

Ciencias $₫$ Agua
2021, Instituto Mexicano de Tecnología del Agua

Open Access bajo la licencia CC BY-NC-SA 4.0

(https://creativecommons.org/licenses/by-nc-sa/4.0/)

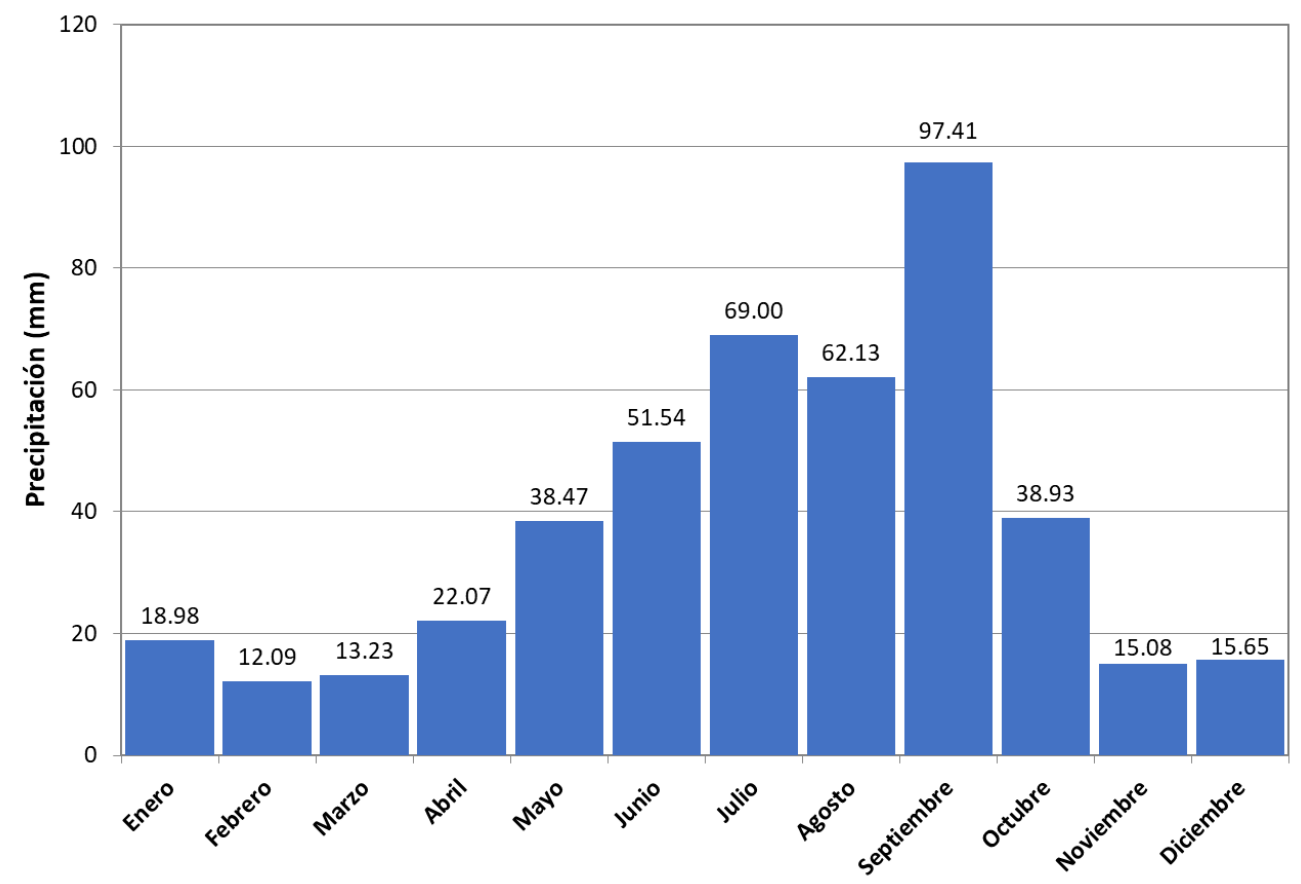

Figura 8. Distribución de la precipitación media mensual en la cuenca del río Bravo.

\section{Identificación de regiones homogéneas ( $R H$ )}

Al inicio del proceso iterativo para la regionalización de la cuenca se partió de la hipótesis de considerar que las 90 estaciones climatológicas seleccionadas pertenecían a una misma y única región homogénea. Sin embargo, esta región originalmente propuesta no superó el criterio de 
homogeneidad de Wallis et al. (2007), pues el valor resultante de $H$ fue de 27.9, es decir, muy superior al valor crítico de referencia que es de 2.0. Entonces, se descartó la idea de considerar a todas las estaciones dentro de una sola región homogénea y se decidió agrupar las estaciones en cinco regiones con base en su PMA; pero al hacer el análisis correspondiente dentro de cada región propuesta se encontraron varias estaciones discordantes del resto. Dichas estaciones se identificaron en cada región mediante una exploración visual a través de los diagramas de ratios de L-CV vs L-asimetría como el que se presenta en la Figura 9. En esta gráfica se aprecia una nube de puntos azules relativamente uniforme respecto a un valor central (cuadro rojo), y en ella es posible identificar aquellas estaciones cuyos L-momentos se apartan más del patrón del resto de las estaciones. Sin embargo, la apreciación gráfica es un método subjetivo, por lo que fue imprescindible calcular los valores $D_{i}$ para cada estación y el estadístico $H$ para cada región. 
Teçnología y

Ciencias $₫$ Agua
2021, Instituto Mexicano de Tecnología del Agua

Open Access bajo la licencia CC BY-NC-SA 4.0

(https://creativecommons.org/licenses/by-nc-sa/4.0/)

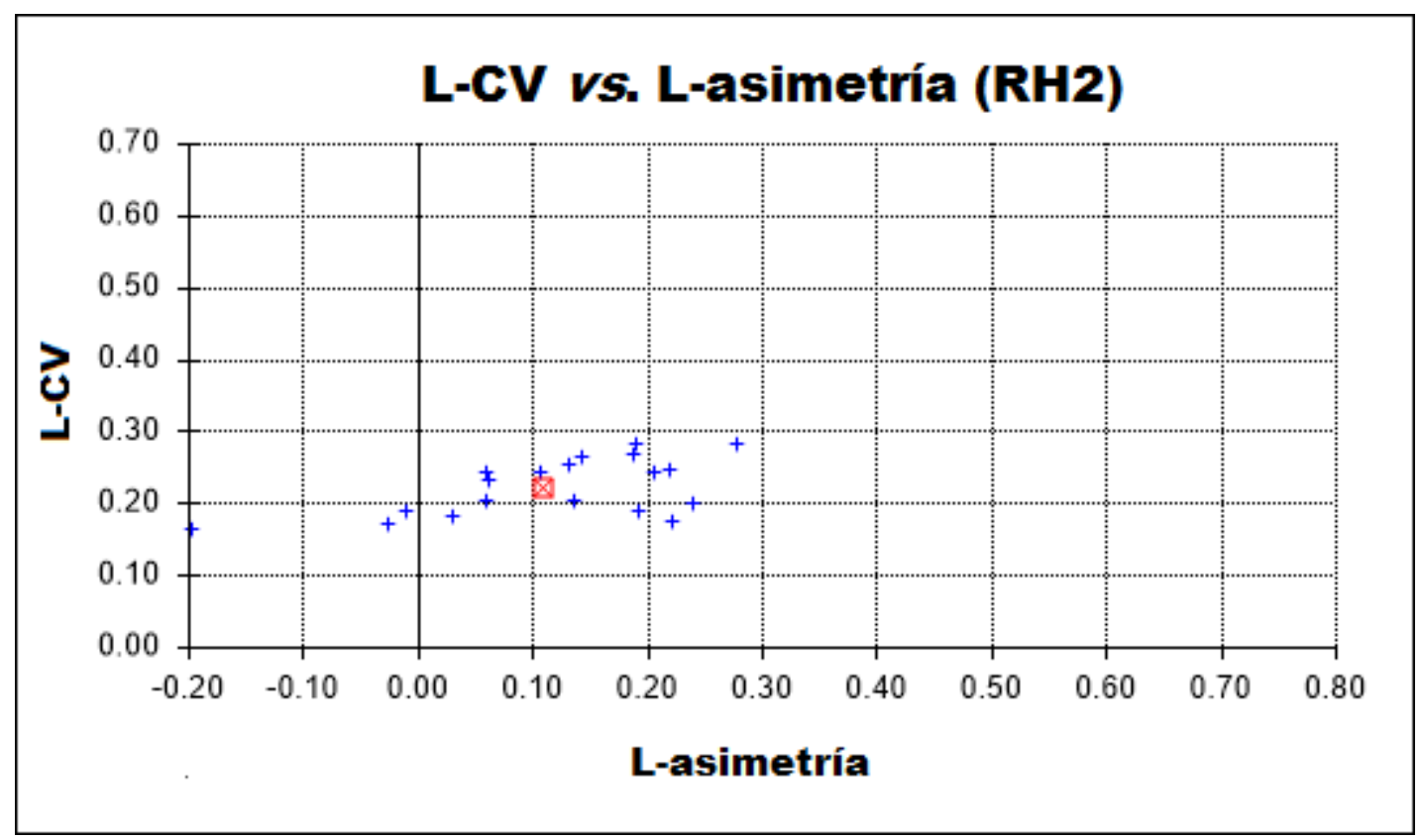

Figura 9. Ejemplo de diagrama de ratios de L-CV vs. L-asimetría para una región determinada.

Así, durante el proceso iterativo, algunas estaciones discordantes se reubicaron en una nueva región, pues presentaban valores de discordancia muy superiores a los valores críticos de referencia. De esta manera, después de varias pruebas para diferente regionalización, quedaron conformadas las cinco regiones siguientes: $\mathrm{RH} 1$ con 24 estaciones y PMA de 256.9 mm; RH2 con 23 estaciones y PMA de 368.8 mm; RH3 con 26 estaciones y PMA de 526.3 mm; RH4 con nueve estaciones y PMA de $675.8 \mathrm{~mm}$; y RH5 con ocho estaciones y PMA de $910.6 \mathrm{~mm}$ (Figura 10). 


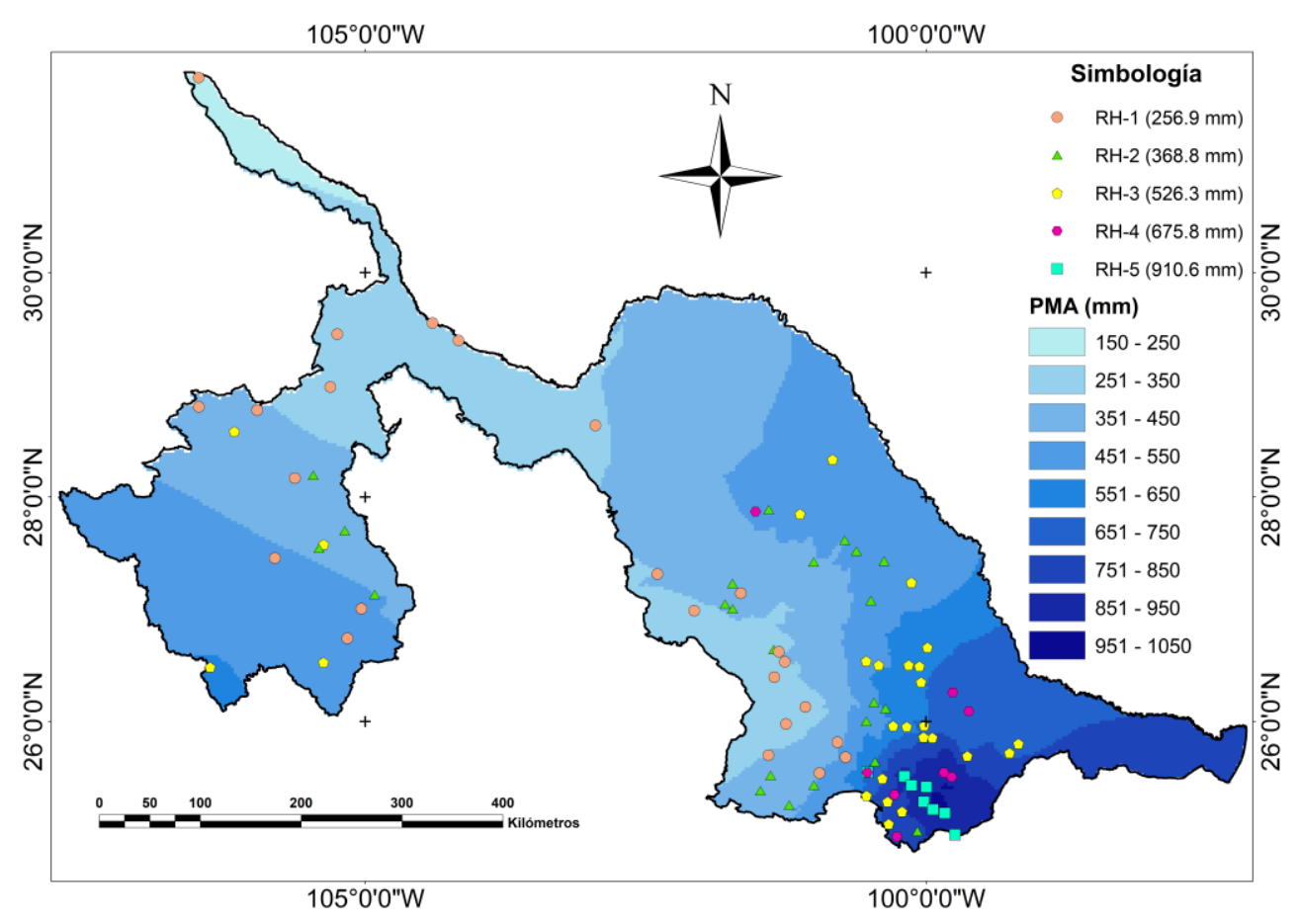

Figura 10. Distribución espacial de las regiones homogéneas con base en el criterio de PMA.

De acuerdo con Hosking y Wallis (1997), las regiones homogéneas no requieren ser geográficamente continuas (tal como se puede observar en la Figura 10), de modo que no se forzó a las estaciones a pertenecer a una determinada región en función de su ubicación geográfica. Así, aunque las estaciones homogéneas tienden a agruparse espacialmente, también sucede que esta condición no se cumple para todas, por lo que además de llamarse "regiones homogéneas", también podrían denominarse "grupos homogéneos" de estaciones, ya que algunas de ellas se ubican lejos de las demás que cumplen con los criterios de 
Tecnología y

Ciencias $₫$ Agua
2021, Instituto Mexicano de Tecnología del Agua

Open Access bajo la licencia CC BY-NC-SA 4.0

(https://creativecommons.org/licenses/by-nc-sa/4.0/)

homogeneidad. En ello son determinantes las condiciones naturales de la cuenca.

Estas regiones cumplieron los criterios de homogeneidad de Hosking y Wallis (1997), y de Wallis et al. (2007), tal como se puede verificar en la Tabla 3 y Tabla 4 , donde se presentan los valores de $D_{i}$ obtenidos para cada estación y los valores del estadístico $H$ en cada región, respectivamente, los cuales, en todos los casos, son inferiores a los valores críticos de referencia. Por lo anterior, se aceptaron estas regiones homogéneas propuestas y se procedió a determinar la distribución de frecuencias de mejor ajuste para cada una de ellas.

Tabla 3. Valores de discordancia $\left(D_{i}\right)$ para cada una de las estaciones por región homogénea.

\begin{tabular}{|c|c|c|c|c|c|c|c|c|c|}
\hline \multicolumn{2}{|c|}{ RH1 } & \multicolumn{2}{|c|}{ RH2 } & \multicolumn{2}{|c|}{ RH3 } & \multicolumn{2}{|c|}{ RH4 } & \multicolumn{2}{|c|}{ RH5 } \\
\hline $\begin{array}{l}\text { Núm. est } \\
D_{i} \text { crítico }=\end{array}$ & $\begin{array}{l}24 \\
.000\end{array}$ & $\begin{array}{l}\text { Núm. est } \\
D_{i} \text { crítico }\end{array}$ & $\begin{array}{l}23 \\
.000\end{array}$ & $\begin{array}{l}\text { Núm. est } \\
D_{i} \text { crítico }\end{array}$ & $\begin{array}{l}.26 \\
.000\end{array}$ & $\begin{array}{c}\text { Núm. est } \\
D_{i} \text { crítico }=\end{array}$ & $\begin{array}{l}=9 \\
2.329\end{array}$ & $\begin{array}{c}\text { Núm. es } \\
D_{i} \text { crítico }\end{array}$ & $\begin{array}{l}=8 \\
2.140\end{array}$ \\
\hline Estación & $\overline{D_{i}}$ & Estación & $\overline{D_{i}}$ & Estación & $\overline{D_{i}}$ & Estación & $\overline{D_{i}}$ & Estación & $\overline{D_{i}}$ \\
\hline 5151 & 0.67 & 8044 & 0.67 & 5002 & 0.57 & 19002 & 0.64 & 19003 & 1.63 \\
\hline 5152 & 0.91 & 5163 & 0.37 & 19024 & 0.09 & 19010 & 1.68 & 19007 & 0.85 \\
\hline 19165 & 0.25 & 19170 & 0.70 & 10141 & 1.96 & 8267 & 2.24 & 19146 & 0.24 \\
\hline 8247 & 1.61 & 5167 & 1.92 & 19009 & 0.89 & 19104 & 0.04 & 19015 & 0.60 \\
\hline 5044 & 0.62 & 5016 & 2.66 & 19012 & 2.32 & 19033 & 1.04 & 19069 & 1.15 \\
\hline 5011 & 1.38 & 8081 & 1.33 & 5149 & 2.63 & 19047 & 1.34 & 19031 & 1.55 \\
\hline 5013 & 0.50 & 19096 & 0.96 & 19013 & 1.36 & 5020 & 1.31 & 19048 & 0.46 \\
\hline
\end{tabular}


Tecnología y

Ciencias $\stackrel{\Xi}{\Im}$ gua
2021, Instituto Mexicano de Tecnología del Agua

Open Access bajo la licencia CC BY-NC-SA 4.0

(https://creativecommons.org/licenses/by-nc-sa/4.0/)

\begin{tabular}{|l|c|c|c|c|c|c|l|l|l|}
\hline 5158 & 0.45 & 5170 & 0.13 & 19105 & 1.12 & 19056 & 0.41 & 19173 & 1.51 \\
\hline 8270 & 0.49 & 5155 & 1.36 & 19117 & 0.77 & 19140 & 0.29 & & \\
\hline 8049 & 0.77 & 19028 & 0.51 & 19016 & 1.08 & & & & \\
\hline 5022 & 0.66 & 19045 & 0.99 & 19018 & 0.14 & & & & \\
\hline 8254 & 0.64 & 5156 & 0.72 & 19021 & 0.43 & & & & \\
\hline 8202 & 2.02 & 5030 & 0.32 & 19022 & 1.02 & & & & \\
\hline 5140 & 2.06 & 5031 & 0.24 & 19124 & 0.49 & & & & \\
\hline 19054 & 0.39 & 19158 & 0.69 & 19042 & 1.06 & & & & \\
\hline 5164 & 0.59 & 19053 & 1.84 & 5148 & 0.38 & & & & \\
\hline 5038 & 2.99 & 5171 & 2.19 & 8185 & 1.59 & & & & \\
\hline 5142 & 0.87 & 19055 & 1.25 & 19131 & 1.40 & & & & \\
\hline 8052 & 0.77 & 5048 & 0.61 & 5033 & 0.12 & & & & \\
\hline 8099 & 2.26 & 5145 & 0.52 & 19133 & 0.18 & & & & \\
\hline 8153 & 1.22 & 5045 & 2.14 & 19134 & 0.74 & & & & \\
\hline 8156 & 0.89 & 8085 & 2.60 & 19178 & 0.92 & & & & \\
\hline 8194 & 1.18 & 8162 & 1.75 & 19063 & 2.39 & & & & \\
\hline 8213 & 1.48 & & & 19141 & 0.34 & & & & \\
\hline & & & & 8322 & 2.74 & & & & \\
\hline & & & & 8311 & 2.72 & & & & \\
\hline
\end{tabular}

Tabla 4. Valores de PMA y $H$ por región homogénea. 


\begin{tabular}{|c|c|c|c|}
\hline $\begin{array}{c}\text { Regiones } \\
\text { Homogéneas }\end{array}$ & PMA & $\boldsymbol{H}$ & $\begin{array}{c}\text { Clasificación } \\
\text { (Wallis et al., 2007) }\end{array}$ \\
\hline RH1 & 256.9 & 1.23 & Homogénea \\
\hline RH2 & 368.8 & 1.39 & Homogénea \\
\hline RH3 & 526.3 & 0.94 & Homogénea \\
\hline RH4 & 675.8 & 1.92 & Homogénea \\
\hline RH5 & 910.6 & -0.63 & Homogénea \\
\hline
\end{tabular}

\section{Selección de la función de distribución de probabilidades (FDP) de mejor ajuste}

Una vez que las $\mathrm{RH}$ propuestas han satisfecho la condición de homogeneidad, es factible determinar la distribución de probabilidad regional y sus parámetros, con base tanto en el uso de los diagramas de los L-momentos-ratios regionales como en el uso de la prueba de bondad de ajuste $Z^{\text {DIST }}$. Para ello, se calcularon los L-momentos regionales, los cuales se muestran en la Tabla 5 para cada una de las RH aceptadas.

Tabla 5. L-momentos regionales para las $\mathrm{RH}$. 
Tecnología y

Ciencias $₫$ Agua
2021, Instituto Mexicano de Tecnología del Agua

Open Access bajo la licencia CC BY-NC-SA 4.0

(https://creativecommons.org/licenses/by-nc-sa/4.0/)

\begin{tabular}{|c|c|c|c|}
\hline \multirow{2}{*}{ Regiones homogéneas } & \multicolumn{3}{|c|}{ Momentos regionales } \\
\cline { 2 - 4 } & L-CV & L-asimetría & L-curtosis \\
\hline $\mathrm{RH} 1$ & 0.237 & 0.053 & 0.097 \\
\hline $\mathrm{RH} 2$ & 0.221 & 0.102 & 0.156 \\
\hline $\mathrm{RH} 3$ & 0.197 & 0.108 & 0.169 \\
\hline $\mathrm{RH} 4$ & 0.205 & 0.077 & 0.148 \\
\hline $\mathrm{RH} 5$ & 0.165 & 0.113 & 0.165 \\
\hline
\end{tabular}

Igualmente, se obtuvieron los diagramas de los L-momentos-ratios para cada región (Figura 11), donde se relacionan los coeficientes de Lasimetría vs. L-curtosis. En estos diagramas se puede apreciar la ubicación del valor de los L-momentos regionales (cuadro rojo) con respecto a las distintas FDP (líneas de colores): GL (azul); LP3 (verde olivo); GEV (verde oscuro); Gaucho (gris oscuro), y GP (rojo), donde se infieren las posibles distribuciones que podrían ajustarse a los datos observados de las estaciones que conforman cada región. 
Tecnología y

Ciencias $₫$ Agua
2021, Instituto Mexicano de Tecnología del Agua

Open Access bajo la licencia CC BY-NC-SA 4.0

(https://creativecommons.org/licenses/by-nc-sa/4.0/)

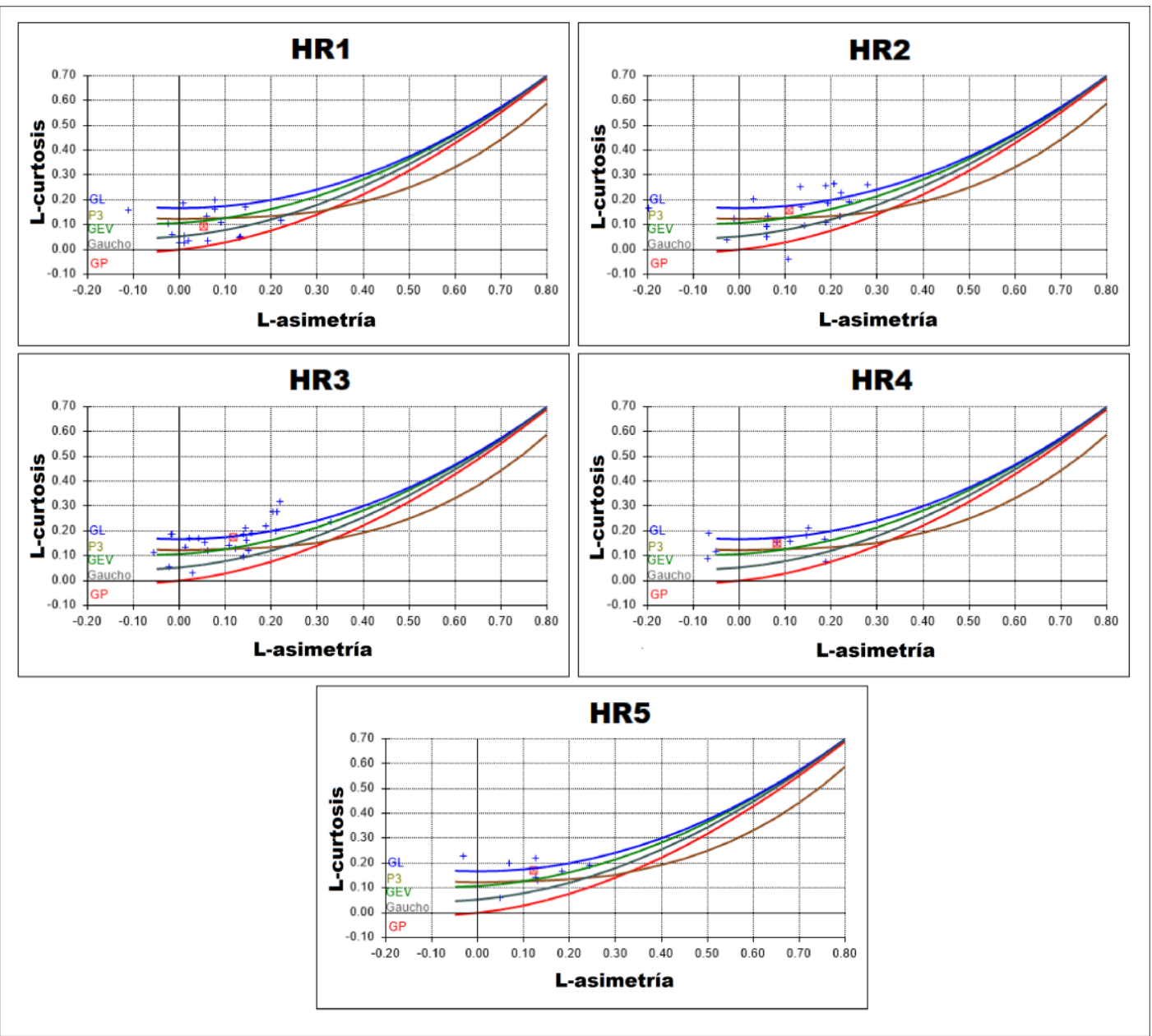

Figura 11. Diagramas de L-momentos-ratios para cada RH.

Según estos diagramas, los L-momentos regionales se comportan de la siguiente manera: en la $\mathrm{RH} 1$, el valor medio se ubica entre las distribuciones GEV y Gaucho; mientras que en las RH 2, 3, 4 y 5 se localiza más cerca de las distribuciones GL y GEV. De este modo, al parecer, cualquiera de tales distribuciones podría representar un modelo adecuado a los datos. Aunque, como señalan Hosking y Wallis (1997), y Peel, Wang, 
Tecnología y

Ciencias $₫$ Agua
2021, Instituto Mexicano de Tecnología del Agua

Open Access bajo la licencia CCBY-NC-SA 4.0

(https://creativecommons.org/licenses/by-nc-sa/4.0/)

Vogel y McMahon (2001), la decisión final no debe depender sólo del análisis de los diagramas de L-momentos-ratios, requiriéndose un método objetivo para la selección adecuada, que en este caso es el estadístico $Z^{D I S T}$.

La Tabla 6 muestra los valores obtenidos de $Z^{\text {DIST }}$ para cada región homogénea; se resaltan en negritas aquellos que son menores al valor de referencia ( $Z^{\text {DIST }} \leq|1.64|$ ). En ella se puede corroborar lo observado en las gráficas anteriores, es decir, que no hay una única FDP que se ajuste a todas las RH. Sin embargo, para la RH1 la FDP de mejor ajuste es la Gaucho, mientras que para las RH 2, 3, 4 y 5 la FDP que coincide como la más adecuada es la GL, por lo cual se eligió esta última. En el Anexo 2 se presentan las fórmulas matemáticas que describen ambas distribuciones (Gaucho y GL).

Tabla 6. Valores de $Z^{\text {DIST }}$ para cada región homogénea.

\begin{tabular}{|c|c|c|c|c|c|c|}
\hline \multirow{2}{*}{$\begin{array}{c}\text { Regiones } \\
\text { homogéneas }\end{array}$} & \multicolumn{5}{|c|}{ Funciones de distribución de probabilidades } \\
\cline { 2 - 7 } & Gaucho & GEV & LP3 & GN & GPA & GL \\
\hline $\mathrm{RH} 1$ & $\mathbf{- 1 . 5 8}$ & 1.95 & 2.50 & 2.62 & -5.13 & 5.68 \\
\hline $\mathrm{RH} 2$ & -4.88 & -1.85 & -2.04 & -1.66 & -8.02 & $\mathbf{1 . 2 1}$ \\
\hline $\mathrm{RH} 3$ & -5.74 & -2.71 & -3.03 & -2.59 & -8.88 & $\mathbf{0 . 3 1}$ \\
\hline $\mathrm{RH} 4$ & -3.29 & $\mathbf{- 1 . 1 6}$ & $\mathbf{- 1 . 0 5}$ & $\mathbf{- 0 . 8 9}$ & -5.46 & $\mathbf{1 . 0 3}$ \\
\hline $\mathrm{RH} 5$ & -3.08 & $\mathbf{- 1 . 3 3}$ & $\mathbf{- 1 . 5 6}$ & $\mathbf{- 1 . 2 8}$ & -4.89 & $\mathbf{0 . 4 0}$ \\
\hline
\end{tabular}


Al identificar la FDP correspondiente a cada región se obtuvieron sus parámetros de ubicación, escala y forma, los cuales se muestran en la Tabla 7. Estos parámetros son de utilidad para determinar los cuantiles para un conjunto de valores de probabilidad.

Tabla 7. Parámetros de la FDP para las regiones homogéneas.

\begin{tabular}{|c|c|c|c|c|c|}
\hline \multirow{2}{*}{$\begin{array}{c}\text { Regiones } \\
\text { homogéneas }\end{array}$} & \multirow{2}{*}{ FDP } & \multicolumn{4}{|c|}{ Parámetros } \\
\cline { 3 - 6 } & & Ubicación & Escala & $\begin{array}{c}\text { Forma } \\
(\mathbf{k})\end{array}$ & $\begin{array}{c}\text { Forma } \\
(\mathbf{h})\end{array}$ \\
\hline $\mathrm{RH} 1$ & Gaucho & 0.6745 & 0.6292 & 0.4408 & 0.500 \\
\hline $\mathrm{RH} 2$ & $\mathrm{GL}$ & 0.9606 & 0.2165 & -0.1091 & -- \\
\hline $\mathrm{RH} 3$ & $\mathrm{GL}$ & 0.9622 & 0.1922 & -0.1176 & -- \\
\hline $\mathrm{RH} 4$ & $\mathrm{GL}$ & 0.9722 & 0.2024 & -0.0829 & -- \\
\hline $\mathrm{RH} 5$ & $\mathrm{GL}$ & 0.9673 & 0.1607 & -0.1217 & -- \\
\hline
\end{tabular}

\section{Estimación de cuantiles}

Los cuantiles son valores asociados con un determinado valor de probabilidad, dependiente de una función de distribución acumulada de probabilidad especificada en sus parámetros. Con las FDP seleccionadas, 
Tecnología y

Ciencias $₫$ Agua
2021, Instituto Mexicano de Tecnología del Agua

Open Access bajo la licencia CC BY-NC-SA 4.0

(https://creativecommons.org/licenses/by-nc-sa/4.0/)

se estimaron los cuantiles para cada una de las regiones homogéneas (Tabla 8), generando así las curvas de crecimiento regional (Figura 12). De esta figura se puede extraer que el periodo de retorno de un año cuya precipitación anual sea, por ejemplo, del $60 \%$ de la PMA $\left(P_{i} / \mathrm{PMA}=0.6\right)$ en la región de la cuenca donde llueve menos (RH1), sería de cinco años, es decir, ese periodo corresponde al tiempo de recurrencia que tendría el evento.

Tabla 8. Cuantiles por región homogénea.

\begin{tabular}{|l|c|c|c|c|c|c|c|c|c|c|c|c|c|c|c|c|c|c|}
\hline \multirow{2}{*}{ RH } & \multirow{2}{*}{ FDP } & \multicolumn{10}{|c|}{ Cuantiles } \\
\cline { 3 - 15 } & & $\mathbf{0 . 0 0 5}$ & $\mathbf{0 . 0 1}$ & $\mathbf{0 . 0 2}$ & $\mathbf{0 . 0 5}$ & $\mathbf{0 . 1}$ & $\mathbf{0 . 2}$ & $\mathbf{0 . 3}$ & $\mathbf{0 . 4}$ & $\mathbf{0 . 5}$ & $\mathbf{0 . 6}$ & $\mathbf{0 . 7}$ & $\mathbf{0 . 8}$ & $\mathbf{0 . 9}$ & $\mathbf{0 . 9 5}$ & $\mathbf{0 . 9 6}$ & $\mathbf{0 . 9 8}$ & $\mathbf{0 . 9 9}$ \\
\hline RH1 & Gaucho & 0.23 & 0.25 & 0.29 & 0.37 & 0.46 & 0.61 & 0.74 & 0.86 & 0.97 & 1.1 & 1.23 & 1.38 & 1.58 & 1.72 & 1.75 & 1.85 & 1.91 \\
\hline RH2 & GL & 0.09 & 0.18 & 0.27 & 0.42 & 0.54 & 0.68 & 0.79 & 0.87 & 0.96 & 1.05 & 1.15 & 1.28 & 1.5 & 1.71 & 1.78 & 2.01 & 2.25 \\
\hline RH3 & GL & 0.21 & 0.28 & 0.36 & 0.48 & 0.59 & 0.72 & 0.81 & 0.89 & 0.96 & 1.04 & 1.13 & 1.25 & 1.44 & 1.64 & 1.7 & 1.91 & 2.13 \\
\hline RH4 & GL & 0.1 & 0.2 & 0.3 & 0.44 & 0.57 & 0.71 & 0.81 & 0.89 & 0.97 & 1.06 & 1.15 & 1.27 & 1.46 & 1.65 & 1.71 & 1.9 & 2.1 \\
\hline RH5 & GL & 0.34 & 0.4 & 0.47 & 0.57 & 0.66 & 0.76 & 0.84 & 0.9 & 0.97 & 1.03 & 1.11 & 1.21 & 1.37 & 1.54 & 1.59 & 1.77 & 1.96 \\
\hline
\end{tabular}




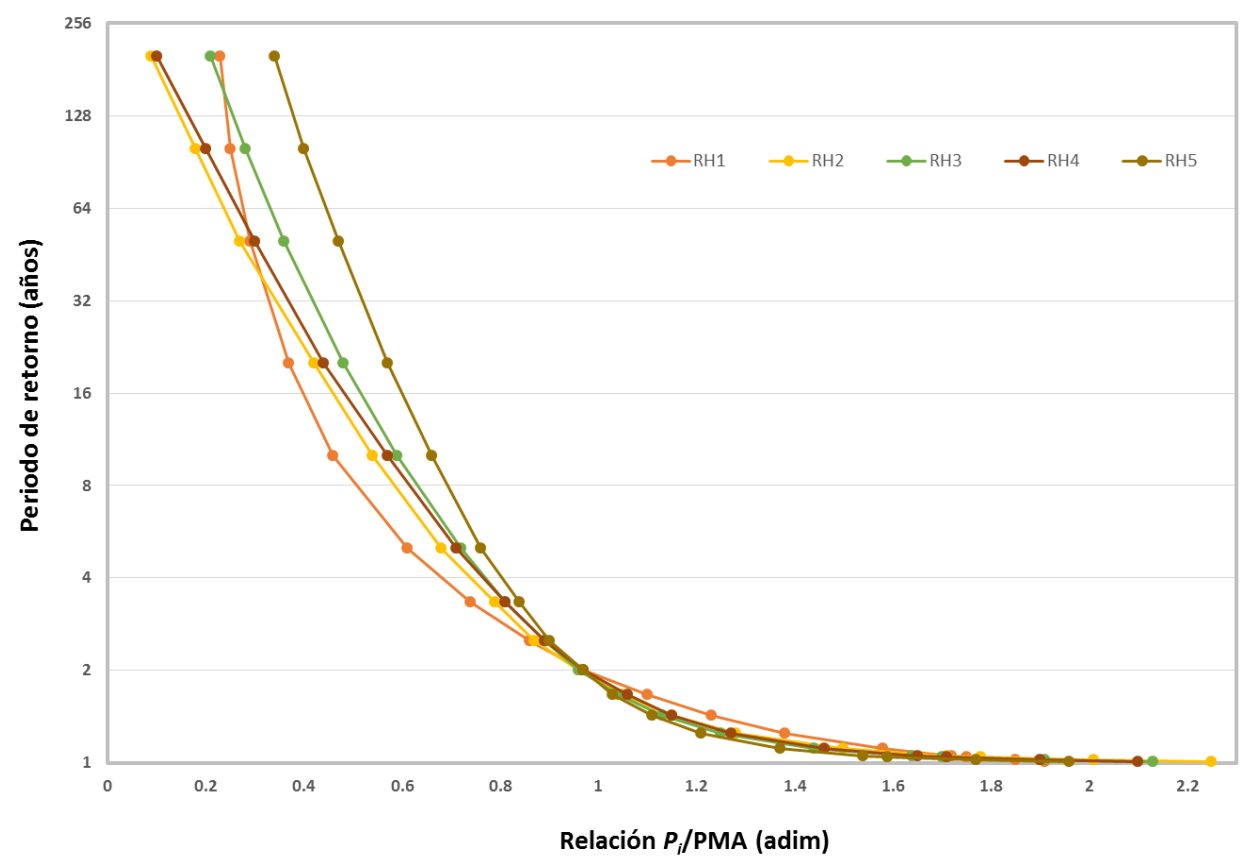

Figura 12. Curvas de crecimiento regional por región homogénea.

Los cuantiles $0.005,0.01,0.02$ y 0.05 están asociados con la presencia de eventos pluviométricos más secos con periodos de retorno de 200, 100, 50 y 20 años, respectivamente. El empleo de los cuantiles junto con los L-momentos y los parámetros de las FDP seleccionadas pueden usarse para determinar de mejor manera la probabilidad de no excedencia de precipitación respecto a la media anual para diferentes periodos de retorno.

De acuerdo con la Tabla 9, para un periodo de retorno ( $T r$ ) de 20 años se espera en la RH1 por lo menos un evento tan bajo como 94.78 mm de la PMA; para el mismo Tr se espera en la RH5 por lo menos un 
Tecnología y

Ciencias $₫$ Agua
2021, Instituto Mexicano de Tecnología del Agua

Open Access bajo la licencia CC BY-NC-SA 4.0

(https://creativecommons.org/licenses/by-nc-sa/4.0/)

evento tan bajo como $518.70 \mathrm{~mm}$ de la PMA. Considerando este periodo a 20 años se puede notar que la RH1 tendría un déficit respecto a su media anual de más del 60 \%; las RH 2, 3 y 4 rondarían en un rango del 50 al $60 \%$ de déficit, mientras que para la RH5 se presentaría el valor más bajo de toda la cuenca con aproximadamente un $43 \%$ de déficit.

Tabla 9. L-momentos y parámetros para cada región homogénea, y la $P_{i}$ para diferentes periodos de retorno.

\begin{tabular}{|c|c|c|c|c|c|c|c|c|c|c|c|c|}
\hline \multirow{2}{*}{$\mathbf{R H}$} & \multirow{2}{*}{ PMA } & \multirow{2}{*}{ FDP } & \multicolumn{4}{|c|}{ Parámetros de FDP } & \multicolumn{6}{|c|}{$\begin{array}{l}\text { Periodos de retorno } \\
\text { (precipitación en } \mathrm{mm} \text { ) }\end{array}$} \\
\hline & & & Ubicación & Escala & $\begin{array}{c}\text { Forma } \\
\text { (k) }\end{array}$ & $\begin{array}{c}\text { Forma } \\
\text { (h) }\end{array}$ & $\operatorname{Tr} 5$ & $\operatorname{Tr} 10$ & $\operatorname{Tr} 15$ & $\operatorname{Tr} 20$ & $\operatorname{Tr} 50$ & $\operatorname{Tr} 100$ \\
\hline $\mathrm{RH} 1$ & 256.9 & Gaucho & 0.6745 & 0.6292 & 0.4408 & 0.500 & 156.6 & 119.0 & 103.6 & 94.7 & 74.5 & 64.8 \\
\hline $\mathrm{RH} 2$ & 368.8 & $\mathrm{GL}$ & 0.9606 & 0.2165 & -0.1091 & ------ & 251.5 & 198.2 & 171.2 & 153.1 & 101.0 & 65.7 \\
\hline $\mathrm{RH} 3$ & 526.3 & $\overline{G L}$ & 0.9622 & 0.1922 & -0.1176 & ------ & 377.0 & 310.5 & 276.9 & 254.6 & 190.5 & 147.3 \\
\hline $\mathrm{RH} 4$ & 675.8 & GL & 0.9722 & 0.2024 & -0.0829 & ------ & 477.8 & 382.2 & 332.8 & 299.6 & 202.0 & 134.3 \\
\hline RH5 & 910.6 & GL & 0.9673 & 0.1607 & -0.1217 & ----- & 694.1 & 598.7 & 550.5 & 518.7 & 427.2 & 365.7 \\
\hline
\end{tabular}

Como se puede observar en la Tabla 9, la intensidad de los episodios de sequía está inversamente relacionada con su frecuencia; es decir, aquellos eventos que son más extremos ocurren con menor frecuencia que los moderados. Asimismo, las sequías afectan en mayor grado a las regiones con niveles de PMA más bajos, obteniéndose, por ejemplo, valores de déficit de $31.8 \%$ para un $\operatorname{Tr}$ de 5 y hasta $82.2 \%$ para un $\operatorname{Tr}$ 
de 100 en la RH2, mientras que para la RH5 en los mismos periodos de retorno los valores de déficit oscilan entre 23.8 y $59.8 \%$.

Lo anterior, considerando únicamente los valores obtenidos por región homogénea. Sin embargo, para determinar la duración y periodicidad promedio (recurrencia) de los periodos secos en cada región, se hizo también un análisis puntual por estación climatológica, donde se determinaron los parámetros de cada una de las estaciones con base en la FDP que le corresponde, por lo que ahora, además de involucrar a todas las estaciones dentro de la misma $\mathrm{RH}$, se hizo el análisis con sus propios parámetros de ubicación, escala y forma, para generar finalmente con ayuda de sus cuantiles los mapas de déficit de precipitación para diferentes periodos de retorno.

\section{Análisis de periodos secos}

Para determinar el tipo de periodo (seco o húmedo) ocurrido durante un año en particular en cada estación climatológica, el umbral que se utilizó como referencia fue la PMA histórica, que en este caso corresponde a la normal climatológica del periodo de 30 años bajo estudio (1984-2013). Así, a partir de los registros de precipitación mensual y anual, se realizó la caracterización de los periodos de sequía meteorológica para cada una de las estaciones dentro de las cinco diferentes regiones homogéneas. 
Tecnología y

Ciencias $₫$ Agua
2021, Instituto Mexicano de Tecnología del Agua

Open Access bajo la licencia CCBY-NC-SA 4.0

(https://creativecommons.org/licenses/by-nc-sa/4.0/)

Después se determinó la duración y periodicidad promedio de los periodos secos, y se identificó el periodo más prolongado y el año más seco en cada región, tal como se muestra en la Tabla 10.

Tabla 10. Características de los periodos secos registrados en cada región homogénea.

\begin{tabular}{|c|c|c|c|c|c|c|c|}
\hline \multirow[b]{2}{*}{ RH } & \multirow{2}{*}{$\begin{array}{c}\text { Número } \\
\text { de años } \\
\text { con } \\
\text { registro }\end{array}$} & \multicolumn{2}{|c|}{ Periodos de sequía } & \multicolumn{2}{|c|}{$\begin{array}{c}\text { Periodo de sequía más } \\
\text { prolongado }\end{array}$} & \multicolumn{2}{|c|}{ Años secos } \\
\hline & & $\begin{array}{c}\text { Duración } \\
\text { promedio } \\
\text { (años) }\end{array}$ & $\begin{array}{c}\text { Periodicidad } \\
\text { promedio } \\
\text { (años) }\end{array}$ & Periodo & $\begin{array}{l}\text { Duración } \\
\text { (años) }\end{array}$ & $\begin{array}{c}\text { Año más } \\
\text { seco }\end{array}$ & $\begin{array}{c}\% \text { respecto } \\
\text { a la media }\end{array}$ \\
\hline $\mathrm{RH} 1$ & 30 & 2.5 & 4.4 & $1998-2002$ & 5 & 2011 & 41.3 \\
\hline $\mathrm{RH} 2$ & 30 & 2.0 & 4.5 & 1993-1996 & 4 & 2011 & 41.6 \\
\hline $\mathrm{RH} 3$ & 30 & 2.0 & 4.4 & $1998-2001$ & 4 & 2011 & 49.8 \\
\hline$\overline{\mathrm{RH}} 4$ & 30 & 2.3 & 4.4 & 1993-1996 & 4 & 2011 & 51.3 \\
\hline RH5 & 30 & 1.7 & 3.9 & $1996-2000$ & 5 & 2011 & 53.9 \\
\hline
\end{tabular}

Con base en esta información se concluye que dentro de la cuenca del río Bravo se presentan eventos de sequía con duración promedio entre 1.7 y 2.5 años, y con una recurrencia media de 3.9 a 4.5 años. De acuerdo con la duración de los periodos de sequía más extensos encontrados en cada una de las regiones homogéneas, se puede afirmar que entre los años 1993 y 2002 ocurrió el periodo de sequía meteorológica más prolongado en la cuenca (con ciertas variaciones de una región a otra). 
Tecnología y

Ciencias $₫$ Agua
2021, Instituto Mexicano de Tecnología del Agua

Open Access bajo la licencia CC BY-NC-SA 4.0

(https://creativecommons.org/licenses/by-nc-sa/4.0/)

Estos resultados concuerdan con los obtenidos por Velasco, Aparicio, Valdéz, Velázquez y Kim (2004), quienes utilizaron el Índice de Precipitación Estandarizado (SPI) y el Índice de Severidad de Sequía de Palmer (PDSI), para evaluar los periodos de sequía meteorológica en las cuencas de afluentes del río Bravo, y determinaron que en el periodo 1993-2001 ocurrió una "pertinaz sequía" en la cuenca del río Conchos, la cual provocó que los volúmenes captados y almacenados en las presas alcanzaran mínimos históricos, ocasionando graves impactos económicos en la actividad agrícola y ganadera de la región. De igual forma, los resultados son similares a los encontrados por Núñez-López, MuñozRobles, Reyes-Gómez, Velasco y Gadsden-Esparza (2007), quienes evaluaron los eventos de sequía meteorológica en el estado de Chihuahua mediante el uso del índice SPI (1970-2004), encontrando que los eventos de sequía más destacados por su intensidad y duración ocurrieron a mediados y finales de la década de 1990, con efectos negativos en la actividad agrícola, en la incidencia de incendios forestales y en el almacenamiento de los embalses más importantes del estado. Asimismo, los resultados coinciden con los reportados por Montero, Santana, Mateos e Ibáñez (2017), quienes analizaron la precipitación extrema en la cuenca del río Conchos (también mediante el índice SPI) para el lapso 1961-2008, y llegaron a la conclusión de que el periodo de sequía de mayor intensidad y duración fue el de 1995-2003.

Debido al fenómeno de propagación de la sequía, el extenso déficit de precipitaciones se traduce en un déficit de escurrimi entos superficiales y en bajos almacenamientos de los embalses; es decir, la sequía meteorológica se convierte en sequía hidrológica (Wilhite \& Glantz, 1985). 
Tecnología y

Ciencias $\stackrel{\Xi}{\Im}$ Aua
2021, Instituto Mexicano de Tecnología del Agua

Open Access bajo la licencia CC BY-NC-SA 4.0

(https://creativecommons.org/licenses/by-nc-sa/4.0/)

Por ello, los resultados anteriores también concuerdan con los reportados por Ortega-Gaucin (2013), quien caracterizó los periodos de sequía hidrológica registrados históricamente en la cuenca del río Bravo, y concluyó que en la mayor parte de la misma ocurrió un periodo de sequía hidrológica extraordinaria que se extendió prácticamente a lo largo de 14 años (1992-2005), el más severo y prolongado del cual se tiene registro. Como consecuencia de esa sequía, el volumen de agua anual utilizado para irrigación en los distritos de riego ubicados en la cuenca (005 Delicias, 090 Bajo Río Conchos y 103 Río Florido, Chihuahua; 004 Don Marín, Coahuila-Nuevo León, y 025 Bajo Río Bravo, Tamaulipas), disminuyó de forma considerable: durante el periodo de 1993-2005 se utilizaron en promedio $1586 \mathrm{hm}^{3}$ de agua para riego, lo cual representó el $64 \%$ de lo que normalmente se empleó en el periodo de 1940-1992 (2 $478 \mathrm{hm}^{3}$ ). Esta situación ocasionó que disminuyera de modo sensible la superficie anual cultivada en dichos distritos de riego: en el periodo 19932005 se regaron en promedio 189710 hectáreas, lo cual representó el 61 \% de la superficie histórica irrigada hasta el año de 1992 (308 537 hectáreas). La sequía extraordinaria también afectó las entregas de agua del río Bravo que México realiza a EUA de acuerdo con el Tratado de 1944, ya que en el periodo de 1993-2005 sólo se pudo entregar en promedio el $60 \%$ de los $432 \mathrm{hm}^{3}$ que se establecen en dicho Tratado como valor medio anual (en total deben entregarse $2158.6 \mathrm{hm}^{3}$ en ciclos de cinco años). Así, desde principios de la década de 1990, México empezó a acumular un déficit en la entrega de aguas del río Bravo a EUA y, conforme a los acuerdos de ambos gobiernos para reducir el déficit, éste se cubrió parcialmente a mediados de la primera década del presente siglo 
y se saldó por completo en el año 2010; pero los incumplimientos en el pago de los adeudos correspondientes al nuevo ciclo de cinco años se repitieron ante la situación de la severa sequía que se vivió en el año 2011 en todo el norte del país.

Precisamente el año 2011 fue el identificado en la presente investigación como el año más seco en todas las regiones de la cuenca (ver Tabla 10), donde se tuvo un déficit de precipitación respecto a la media que varió de 41.3 a 53.9 \% de una región a otra. Esto coincide con lo reportado ese año por el Servicio Meteorológico Nacional (SMN) a través del Monitor de Sequía de México (el cual se basa en el análisis e interpretación de diversos índices de sequía meteorológica e hidrológica), cuando el $80 \%$ del territorio nacional estuvo en condiciones de sequía severa, y la región norte en condiciones de sequía excepcional (OrtegaGaucin \& Velasco, 2013). Tan sólo en ese año se perdieron en el país más de 800 mil hectáreas de diferentes cultivos, lo que representó una pérdida aproximada de \$7 750 millones de pesos; en la ganadería, actividad intrínsecamente relacionada con la agricultura, se perdieron cerca de cerca de 1.3 millones de cabezas de ganado, sobre todo bovinos (Cenapred, 2012), lo cual es un indicativo de la gravedad de esa sequía.

A partir de la información obtenida para cada año del registro histórico analizado, se determinaron los diferentes grados de sequía presentes en los distintos periodos secos a lo largo de la cuenca del río Bravo. Para ello se propuso una clasificación del grado de sequía de acuerdo con quintiles (Tabla 11). 
Tecnología y

Ciencias ฐAgua
2021, Instituto Mexicano de Tecnología del Agua

Open Access bajo la licencia CCBY-NC-SA 4.0

(https://creativecommons.org/licenses/by-nc-sa/4.0/)

Tabla 11. Clasificación del grado de sequía meteorológica con base en quintiles.

\begin{tabular}{|c|c|}
\hline Déficit respecto a la media (\%) & Grado de sequía \\
\hline$\leq 20$ & Ligera \\
\hline$>20-40$ & Moderada \\
\hline$>40-60$ & Severa \\
\hline$>60-80$ & Extrema \\
\hline$>80$ & Excepcional \\
\hline
\end{tabular}

Para este análisis se consideró a la lámina disponible ante el déficit por periodo seco, la cual es comparada respecto a la media de la región homogénea, para así asignarle algún grado de sequía como se muestra en la Tabla 12.

Tabla 12. Periodos secos y grados de sequía por región homogénea.

\begin{tabular}{|c|c|c|r|r|r|r|}
\hline RH & $\begin{array}{c}\text { Periodos } \\
\text { secos }\end{array}$ & $\begin{array}{c}\text { Déficit } \\
\text { acumulado } \\
(\mathbf{m m})\end{array}$ & $\begin{array}{c}\text { Intensidad } \\
\mathbf{( m m / a n ̃ o )}\end{array}$ & $\begin{array}{c}\text { Lámina } \\
\text { disponible } \\
\text { ante } \\
\text { déficit } \\
\mathbf{( m m )}\end{array}$ & $\begin{array}{c}\text { Déficit } \\
\text { respecto a } \\
\text { la media } \\
\mathbf{( \% )}\end{array}$ & $\begin{array}{c}\text { Grado de } \\
\text { sequía }\end{array}$ \\
\hline \multirow{2}{*}{$\mathrm{RH} 1$} & $1989-1989$ & 77.4 & 77.4 & 179.6 & 30.1 & Moderada \\
\cline { 2 - 7 } & $1993-1996$ & 202.1 & 50.5 & 54.8 & 78.6 & Extrema \\
\hline
\end{tabular}


Tecnología y

Ciencias $\approx$ Agua
2021, Instituto Mexicano de Tecnología del Agua

Open Access bajo la licencia CC BY-NC-SA 4.0

(https://creativecommons.org/licenses/by-nc-sa/4.0/)

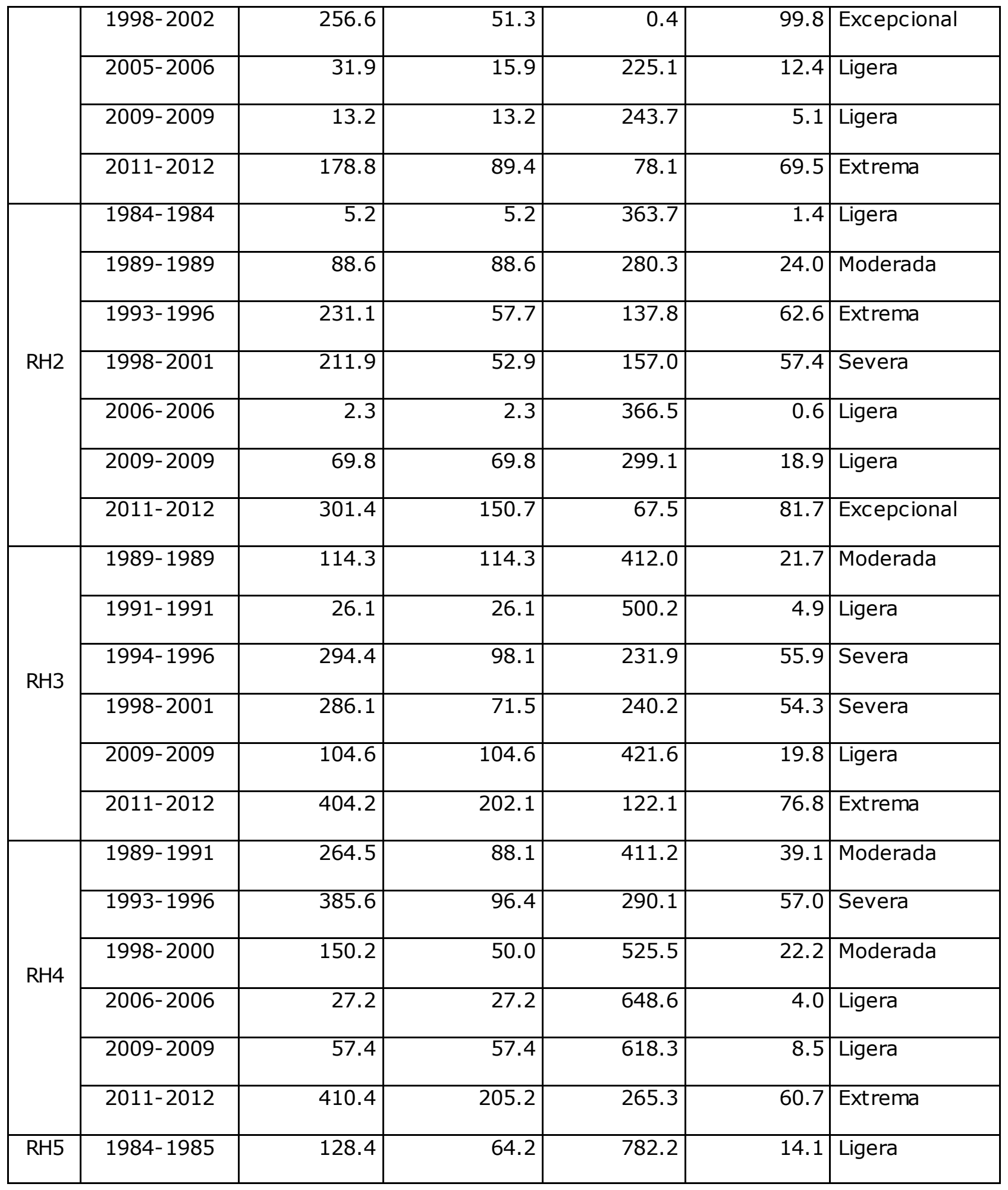


Tecnología y

Ciencias $₫$ Agua
2021, Instituto Mexicano de Tecnología del Agua

Open Access bajo la licencia CC BY-NC-SA 4.0

(https://creativecommons.org/licenses/by-nc-sa/4.0/)

\begin{tabular}{|r|r|r|r|r|r|l|}
\hline $1989-1989$ & 105.8 & 105.8 & 804.8 & 11.6 & Ligera \\
\cline { 2 - 7 } & $1991-1991$ & 6.3 & 6.3 & 904.3 & 0.6 & Ligera \\
\hline $1994-1994$ & 87.8 & 87.8 & 822.7 & 9.6 & Ligera \\
\hline $1996-2000$ & 503.2 & 100.6 & 407.4 & 55.2 & Severa \\
\hline $2006-2006$ & 101.1 & 101.1 & 809.5 & 11.1 & Ligera \\
\hline $2009-2009$ & 105.5 & 105.5 & 805.0 & 11.5 & Ligera \\
\hline
\end{tabular}

Al analizar la Tabla 12 se puede constatar que el periodo 20112012, considerado en otras investigaciones como uno de los eventos de sequía más severos de los últimos años (Ortega-Gaucin \& Velasco, 2013; Arreguín, López, Korenfeld, \& Ortega-Gaucin, 2016b), se encuentra presente en este estudio prácticamente en todas las regiones de la cuenca, con una intensidad que varía de una región a otra, y va desde sequía severa (con déficit de 40-60 \% de la media) hasta sequía excepcional (cuyo déficit alcanza más del $80 \%$ de la media). Los autores citados mencionan que entre los estados más afectados por la sequía ocurrida en el país en ese periodo se encuentran Chihuahua y Coahuila, que se ubican en la parte centro y occidente de la cuenca del río Bravo y pertenecen a las $\mathrm{RH} 1$ y $\mathrm{RH} 2$ generadas en este estudio. Ese periodo seco fue el detonador para que se pusiera en marcha en México el Programa Nacional contra la Sequía (Pronacose), el cual tenía como objetivo implementar un cambio paradigmático en la manera de atender la sequía, al pasar de la reacción frente a las emergencias hacia un modelo preventivo orientado a la gestión del riesgo. Los fundamentos de este 
programa - prevención y mitigación-constituyeron las bases sobre las cuales se buscaba mejorar la gestión versus las sequías y fortalecer las capacidades locales para afrontar sus impactos con estrategias adecuadas (Arreguín et al., 2016b).

\section{Mapeo}

Para una mejor comprensión e interpretación de la magnitud y distribución espacio-temporal de la sequía meteorológica asociada con diferentes periodos de retorno (5, 10, 15, 20, 50 y 100 años), se generaron mapas de déficit de precipitación por cada periodo de retorno en la cuenca del río Bravo (Figura 13). 
Tecnología y

Ciencias $\approx$ Agua
2021, Instituto Mexicano de Tecnología del Agua

Open Access bajo la licencia CC BY-NC-SA 4.0

(https://creativecommons.org/licenses/by-nc-sa/4.0/)
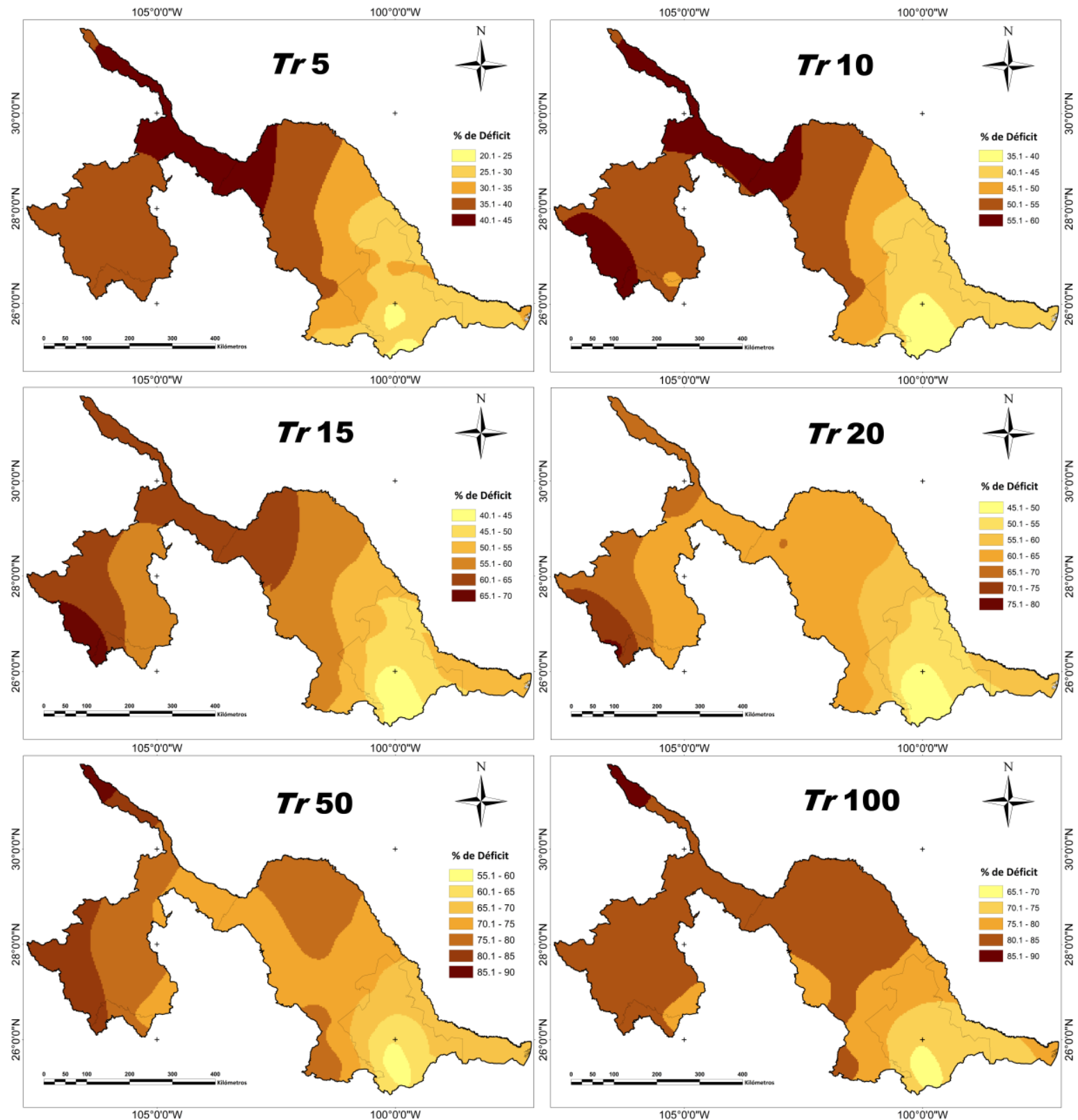

Figura 13. Mapas de déficit de precipitación en la cuenca del río Bravo, México, para diferentes periodos de retorno $(T r)$. 
Tecnología y

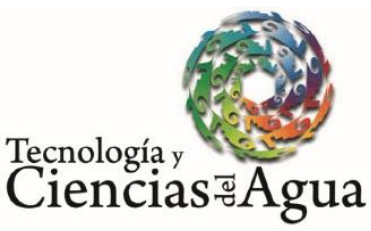

2021, Instituto Mexicano de Tecnología del Agua

Open Access bajo la licencia CCBY-NC-SA 4.0

(https://creativecommons.org/licenses/by-nc-sa/4.0/)

Como se aprecia en los diferentes mapas de la Figura 13, las regiones que presentarían sequía meteorológica con mayor grado déficit de lluvia y menor periodo de retorno serían las $\mathrm{RH} 1$ y $\mathrm{RH} 2$ (las cuales abarcan la mayor parte de la cuenca ubicada en los estados de Chihuahua y Coahuila), en donde los niveles de déficit de la precipitación variarían a lo largo del tiempo hasta presentar niveles por arriba del $80 \%$ de déficit; ello indicaría que para un periodo de retorno de 100 años, más de la mitad de la cuenca presentaría un grado de sequía extraordinaria, lo que podría repercutir de forma significativa en las distintas actividades económicas y en la población.

Estos resultados se relacionan con lo mencionado por Núñez-López et al. (2013), quienes señalan que la proximidad o lejanía de las zonas de costa refleja un claro efecto de continentalidad, indicando una disminución de los valores de precipitación conforme más lejana está la zona marítima. De acuerdo con esto, las RH4 y RH5, que se encuentran en la parte este de la cuenca (en los estados de Tamaulipas y Nuevo León) son aquellas que presentan los niveles más altos de precipitación (601$750 \mathrm{~mm}$ y 751-1000 mm, respectivamente), y por lo obtenido en los mapas generados en este trabajo, la RH5 es la región menos propensa a sufrir un grado extremo de sequía meteorológica, pues la precipitación tiende a incrementarse en valores de longitud próximos al este de la cuenca (costa del Golfo de México), además de que con frecuencia se ve beneficiada por los ciclones tropicales provenientes del Atlántico.

Los grados de sequía que se muestran en los mapas de déficit de precipitación generados se intensifican conforme se incrementa el periodo de retorno, llegando a presentar sequías extremas a excepcionales con 
hasta un $90 \%$ de déficit en la precipitación para el periodo de retorno de 100 años. Esto coincide con los resultados reportados por Kim, Valdés y Aparicio (2002), quienes investigaron las características de las sequías en la cuenca del río Conchos mediante el índice PDSI, encontrando que la sequía más severa (ocurrida en la década de 1990) tiene un periodo de retorno asociado de aproximadamente 80 a 100 años con grandes áreas cubiertas. Esto concuerda con los resultados expuestos por Cerano, Villanueva, Valdez, Méndez y Constante (2011), quienes emplearon métodos dendríticos para reconstruir los registros de precipitación de los últimos 600 años en la región este de la cuenca del río Bravo (en las sierras de Coahuila y Nuevo León), y entre sus conclusiones se encuentra la confirmación de la ocurrencia de sequías y mega sequías con una periodicidad aproximada de 50 a 100 años, las cuales coinciden con registros históricos de los daños sociales y económicos que dichas sequías ocasionaron en México. Por ejemplo, la sequía del "año del hambre" en 1786, y las sequías previas a los movimientos sociales de la Independencia en 1810 y la Revolución en 1910, que influyeron en su desencadenamiento (Florescano, 2000).

\section{Conclusiones}


Este trabajo describió los fundamentos y la aplicación del análisis regional de frecuencia de sequías basado en L-momentos para determinar las características de los periodos secos ocurridos en la cuenca del río Bravo durante el lapso 1984-2013.

Con respecto al método utilizado, se concluye que es una buena alternativa para abordar el análisis de la sequía meteorológica en regiones áridas y semiáridas, como la cuenca del río Bravo, que se caracterizan por tener una gran variabilidad espacial y temporal de las precipitaciones, con influencia de factores externos en la variabilidad decadal, y con limitada disponibilidad de registros de lluvia, pues permite compensar la carencia de información en el tiempo por su abundancia en el espacio. Además, los modelos de distribución de tres o más parámetros utilizados por este método (como la distribución Kappa de cuatro parámetros) son más generales y flexibles que los modelos convencionales usados en la hidrología probabilística, los cuales suelen ser de uno o dos parámetros. Ello permite que ante la presencia de valores atípicos - que pueden tener base física, como precipitaciones anuales atribuidas al fenómeno ENSO, por ejemplo- los ajustes mediante modelos convencionales puedan dar resultados insatisfactorios; pero los L-momentos son muy insensibles a esos valores atípicos, por lo que son más robustos estadísticamente hablando.

Por otro lado, con relación a los resultados obtenidos, el desarrollo de la metodología planteada permitió identificar cinco regiones climáticas homogéneas de acuerdo con la precipitación media anual. La función de distribución de probabilidades que mejor se ajustó a los registros de las estaciones climatológicas en la mayoría de las regiones ( $\mathrm{RH} 2,3,4$ y 5) 
fue la Logística Generalizada (GL), mientras que para la RH1 fue la función de distribución Gaucho. Con base en estas distribuciones se determinaron los cuantiles (valores de precipitación anual asociados con un determinado valor de probabilidad de ocurrencia) para los periodos de retorno de $5,10,15,20,50$ y 100 años.

El análisis de los periodos secos permitió reconocer que en la cuenca del río Bravo se presentan eventos de sequía meteorológica con duración promedio entre 1.7 y 2.5 años, y con una periodicidad promedio (recurrencia) de 3.9 a 4.5 años. De acuerdo con la duración de los periodos de sequía más extensos encontrados en cada una de las regiones homogéneas, se puede afirmar que entre los años 1993 y 2002 ocurrió el periodo de sequía meteorológica más prolongado en la cuenca (con ciertas variaciones de una región a otra), y que el periodo 2011-2012 fue el que presentó mayor déficit de precipitación dentro del lapso estudiado. Esto coincide con otras investigaciones, que señalan a este último periodo seco como el más severo que ha ocurrido en el país en las últimas siete décadas.

Finalmente, se generaron mapas de probabilidad de ocurrencia de sequías con base en su déficit de la precipitación esperada respecto a la media anual para los periodos de retorno mencionados arriba, donde se observa una relación directamente proporcional entre el grado de sequía y la duración del periodo. Las regiones más afectadas de la cuenca para los distintos periodos de retorno son las que se ubican en los estados de Chihuahua y Coahuila, en donde los niveles de déficit de la precipitación pueden variar a lo largo del tiempo hasta presentar niveles superiores al $80 \%$ de déficit, lo que indicaría que para un periodo de retorno de 100 
años, más de la mitad de la cuenca podría presentar un grado de sequía extraordinaria, lo cual tendría graves consecuencias en la disponibilidad del recurso hídrico para los diversos sectores económicos y la población.

Los periodos de retorno estimados en este estudio son útiles como herramienta de planeación tanto para el diseño como para la gestión de los recursos hídricos, sobre todo para plantear escenarios de disponibilidad y uso del agua en una región con severos problemas en la relación oferta/demanda de agua. No obstante, la solución de la problemática de la cuenca debe incluir, de forma ineludible, la generación de políticas públicas binacionales, con el propósito de lograr un ordenamiento territorial y un crecimiento demográfico planeado, así como hacer un uso eficiente, racional y sustentable de los escasos recursos hídricos. Estas acciones son muy importantes para el futuro de la gestión del agua, así como para el cumplimiento del tratado de distribución de aguas de 1944 entre México y EUA. De lo contrario, es previsible que los conflictos entre usuarios por el agua, tanto en las partes alta y baja de la cuenca como en ambos lados de la frontera, se exacerben como consecuencia de la limitada disponibilidad del recurso.

\section{Agradecimientos}

Este artículo forma parte de los trabajos realizados en el proyecto de investigación PN-2017/4924, financiado por el Consejo Nacional de Ciencia y Tecnología (Conacyt) a través del Programa de Proyectos de Desarrollo Científico para Atender Problemas Nacionales. Los autores 
agradecen las observaciones y recomendaciones de tres revisores anónimos que permitieron mejorar el trabajo.

\section{Referencias}

Abolverdi, J., \& Khalili, D. (2010). Probabilistic analysis of extreme regional meteorological droughts by L-moments in a semi-arid environment. Theoretical and Applied Climatology, 102, 351-366.

Acuña, J., Felipe, O., \& Fernández, C. (2015). Análisis regional de frecuencia de precipitación anual para la determinación de mapas de sequías en las cuencas Chillón, Rímac, Lurín y Alto Mantaro. Revista Peruana Geo-Atmosférica RPGA, 4, 93-108.

Acuña, J., Felipe, O., Ordoñez, J., \& Arboleda, F. (2011). Análisis regional de frecuencia de precipitación anual para la determinación de mapas de sequías. Revista Peruana Geo-Atmosférica RPGA, 3, 104-115.

Alexandersson, H. (1986). A homogeneity test applied to precipitation data. Journal of Climatology, 6, 661-675.

Arreguín, F. I., López, M., Korenfeld, D., \& Ortega-Gaucin, D. (2016a). The National Drought Policy in Mexico. Journal of Energy Challenges and Mechanics, 3(3), 157-166.

Arreguín, F. I., López, M., Ortega-Gaucin, D., \& Ibáñez, O. (2016b). La política pública contra la sequía en México: avances, necesidades y perspectivas. Tecnología y ciencias del agua, 7(5), 63-76. 
Arreguín, F. I., López, M., Velázquez, C., \& López, R. (2013). Análisis de sequías en el marco del Tratado sobre Aguas Internacionales de 1944. Tecnología y ciencias del agua, 4(1), 117-148.

Báez, R., Prieto, D. V., \& Aroche, R. (2016). Estudio de aplicación del análisis regional de frecuencias basado en L-momentos al caso de las precipitaciones anuales en la provincia de Camagüey, Cuba. Revista Brasileira de Meteorología, 31(4), 539-545.

Bass, S., Ramasamy, S., Dey-Deprick, J., \& Batista, F. (2008). Disaster risk management systems analysis. Rome, Italy: Food and Agriculture Organization.

Brito-Castillo, L., Vivoni, E. R., Gochis, D. J., Filonov, A., Tereshchenko, L., \& Monzon, C. (2010). An anomaly in the occurrence of the month of maximum precipitation distribution in northwest Mexico. Journal of Arid Environments, 74, 531-539.

Campos-Aranda, D. F. (2014). Análisis regional de frecuencia de crecientes en la región hidrológica no. 10 (Sinaloa), México. 2. Contraste de predicciones locales y regionales. Agrociencia, 48(3), 255-270.

Carrao, H., Naumann, G., \& Barbosa, P. (2016). Mapping global pattems of drought risk: An empirical framework based on sub-national estimates of hazard, exposure and vulnerability. Global Environmental Change, 39, 108-124.

Castillo, C., \& Ortiz, N. (2015). Regionalización de caudales máximos en la cuenca del río Sinú por medio del método estadístico índice de 
creciente (Proyecto de Pregrado). Bogotá, Colombia: Universidad Santo Tomás, Facultad de Ingeniería Ambiental.

Cenapred, Centro Nacional de Prevención de Desastres. (2012). Características e impacto socioeconómico de los principales desastres ocurridos en la república mexicana en el año 2011. México, DF, México: Secretaría de Gobernación.

Cerano, J., Villanueva, J., Valdez, R. D., Méndez, J., \& Constante, V. (2011). Sequías reconstruidas en los últimos 600 años para el noreste de México. Revista Mexicana de Ciencias Agrícolas, Pub. Esp. No. 2, 235-249.

Conagua, Comisión Nacional del Agua. (2018). Estadísticas del agua en México. Ciudad de México, México: Secretaría del Medio Ambiente y Recursos Naturales.

Conagua, Comisión Nacional del Agua. (2016). Atlas del agua en México. Ciudad de México, México: Secretaría del Medio Ambiente y Recursos Naturales.

Conagua, Comisión Nacional del Agua. (2014). Programa de Medidas Preventivas y de Mitigación de la Sequía del Consejo de Cuenca Río Bravo. México, DF, México: Secretaría del Medio Ambiente y Recursos Naturales.

Conagua, Comisión Nacional del Agua. (2010). Estadísticas del agua en México. México, DF, México: Secretaría del Medio Ambiente y Recursos Naturales.

De-la-Cruz, J., \& Ortega-Gaucin, D. (18-20 septiembre, 2019). Análisis regional de frecuencia de sequía meteorológica en la zona árida de 
México. Artículo COMEII-19011. En: Memorias del Quinto Congreso Nacional de Riego y Drenaje COMEII-AURPAES 2019. Mazatlán, Sinaloa, México.

Eslamian, S., Hassanzadeh, H., Abedi-Koupai, J., \& Gheysari, M. (2012). Application of L-moments for regional frequency analysis of monthly drought indexes. Journal of Hydrologic Engineering, 17(1), 32-42.

Esparza, M. (2014). La sequía y la escasez de agua en México. Situación actual y perspectivas futuras. Secuencia, 89, 195-219.

Florescano, E. (2000). Breve historia de la sequía en México, $2^{\mathrm{a}}$ ed. México, DF, México: Consejo Nacional para la Cultura y las Artes.

Greenwood, J. A., Landwehr, J. M., Matalas, N. C., \& Wallis, J. R. (1979). Probability weigthed moments: Definition and relation to parameters of several distributions expressable in inverse form. Water Resources Research, 15, 1049-1054.

Guijarro, J. A. (2018). Homogeneización de series climáticas con Climatol (Versión 3.1.1). Islas Baleares, España: Agencia Estatal de Meteorología.

Hallack, M. \& Ramírez, J. (2010). Estudio de caso de la región noroeste de México: Sonora y Baja California. En: UNESCO (ed.). Guía metodológica para la aplicación del análisis regional de frecuencia de sequías basado en L-momentos y resultados de aplicación en América Latina (77 pp.) (Documentos Técnicos del PHI-LAC, No 27). Montevideo, Uruguay: Centro del Agua para Zonas Áridas y Semiáridas de América Latina y el Caribe. 
Hosking, J. R. M., \& Wallis, J. R. (1997). Regional frequency analysis: An approach based on L-moments. Cambridge, UK: Cambridge University Press.

Kalma, J. and Franks, S. (2003). Rainfall in arid and semiarid regions. In: Simmers, I. (ed.). Understanding water in a dry environment (pp. 15-63). Lisse, The Netherlands: Balkema.

Kim, T. W., Valdés, J. B., \& Aparicio, J. (2002). Frequency and spatial characteristics of droughts in the Conchos River Basin, Mexico. Water International, 27(3), 420-430.

La-Cruz, F. J. (2015). Análisis de las sequías meteorológicas en Venezuela utilizando el método L-momentos (tesis de Doctorado en Ingeniería). Bárbula, Venezuela: Universidad de Carabobo.

Linares, M. (2004). La sequía en la cuenca del río Bravo: principios de política. Gaceta Ecológica, 70, 57-66.

Magaña, V. O., Vázquez, J. L., Pérez, J. L., \& Pérez, J. B. (2003). Impact of El Niño on precipitation in Mexico. Geofísica Internacional , 42(3), 313-330.

Martínez, P. F. (ed.) (2018). La cuenca del río Bravo y el cambio climático. San Andrés Cholula, México: Universidad de las Américas Puebla.

Montero, M., Santana, J. C., Mateos, E., \& Ibáñez, O. F. (2017). Análisis de precipitación extrema para la cuenca del río Conchos, usando el Índice Normalizado de Precipitación. En: Montero, M., \& Ibáñez, O. F. (coords.). La cuenca del río Conchos: una mirada desde las ciencias ante el cambio climático (pp. 85-107). Jiutepec, México: Instituto Mexicano de Tecnología del Agua. 
Naranjo, R. C. A. (2011). Análisis regional de frecuencia mediante el método de los L-momentos en las regiones de Valparaíso y Metropolitana para la generación de cartografía probabilística de sequía meteorológica (Memoria de Título de Ingeniería en Recursos Naturales Renovables). Santiago de Chile, Chile: Universidad de Chile.

Norbiato, D., Borga, M., Sangati, M., \& Zanon, F. (2007). Regional frequency analysis of extreme precipitation in the eastern Italian Alps and the August 29, 2003, flash flood. Journal of Hydrology, 345, 149166.

Núñez-Galeano, L., \& Giraldo-Osorio, J. D. (2016). Adaptation of the Lmoments method for the regionalization for maximum annual temperatures in Colombia. Ingeniería y Universidad, 20(2), 373-389.

Núñez-López, D., Treviño-Garza, E. J., Reyes-Gómez, V. M., MuñozRobles, C. A., Aguirre-Calderón, O. A., \& Jiménez-Pérez, J. (2013). Interpolación espacial de la precipitación media mensual en la cuenca del río Bravo/Grande. Tecnología y ciencias del agua, 4(2), 185-193.

Núñez-López, D., Muñoz-Robles, C. A., Reyes-Gómez, V. M., Velasco, I., \& Gadsden-Esparza, H. (2007). Caracterización de la sequía a diversas escalas de tiempo en Chihuahua, México. Agrociencia, 41(3), 253-262.

Oliver, M. A., \& Webster, R. (1990). Kriging: A method of interpolation for geographic information systems. International Journal of Geographical Information Systems, 4(3), 313-332. 
OMM, Organización Meteorológica Mundial. (2006). Vigilancia y alerta temprana de la sequía: conceptos, progresos y desafíos futuros. OMM-N 1006. Ginebra, Suiza: Organización Meteorológica Mundial. OMM-GWP, Organización Meteorológica Mundial y Asociación Mundial para el Agua. (2016). Manual de indicadores e índices de sequía. Ginebra, Suiza: Organización Meteorológica Mundial y Asociación Mundial para el Agua.

Ortega-Gaucin, D. (2013). Caracterización de las sequías hidrológicas en la cuenca del río Bravo, México. Terra Latinoamericana, 31(3), 167180.

Ortega-Gaucin, D., \& Velasco, I. (2013). Aspectos socioeconómicos y ambientales de las sequías en México. Aqua-LAC, 5(2), 90-90.

Ortega-Gaucin, D., De-la-Cruz, J., \& Castellano, H. V. (2018). Peligro, vulnerabilidad y riesgo por sequía en el contexto del cambio climático en México. En: Lobato, R., \& Pérez, A. (coords.). Agua y cambio climático (pp. 78-103). Jiutepec, México: Instituto Mexicano de Tecnología del Agua.

Paredes, F., La-Cruz, F., \& Guevara, E. (2014). Análisis regional de frecuencia de las sequías meteorológicas en la principal región cerealera de Venezuela. Bioagro, 26(1), 21-28.

Paulhus, J. H. L., \& Kohler, M. A. (1952). Interpolation of missing precipitation records. Monthly Weather Review, 80(8), 129-133.

Peel, M. C., Wang, Q., Vogel, R., \& McMahon, T. (2001). The utility of Lmoment ratio diagrams for selecting a regional probability distribution. Hidrologycal Sciences Journal, 46(1), 147-155. 
Tecnología y

Ciencias $₫$ Agua
2021, Instituto Mexicano de Tecnología del Agua

Open Access bajo la licencia CC BY-NC-SA 4.0

(https://creativecommons.org/licenses/by-nc-sa/4.0/)

Rodríguez, B., \& Pineda-Martínez, L. F. (2017). Análisis de la variabilidad de las precipitaciones en la región transfronteriza del centro-norte de México y el sur de los Estados Unidos. En: Montero, M., \& Ibáñez, O. F. (coords.). La cuenca del río Conchos: una mirada desde las ciencias ante el cambio climático (pp. 59-82). Jiutepec, México: Instituto Mexicano de Tecnología del Agua.

Schaefer, M. G., Barker, B. L., Taylor, G. H., \& Wallis, J. R. (2007). Regional precipitation-frequency analysis and spatial mapping for 24hour precipitation for Oregon. Final Report SPR656. Washington, DC, USA: MGS Engineering Consultants.

Seager, R., Ting, M., Davis, M., Cane, M., Naik, N., Nakamura, J., Li, C., Cook, E., \& Stahle, D. W. (2009). Mexican drought: An observational modeling and tree ring study of variability and climate change. Atmósfera, 22(1), 1-31.

Semarnat, Secretaría de Medio Ambiente y Recursos Naturales. (2 de junio, 2011). Acuerdo por el que se da a conocer el resultado de los estudios técnicos de la Región Hidrológica número 24 BravoConchos. Diario Oficial de la Federación. México, DF, México: Secretaría de Medio Ambiente y Recursos Naturales.

Stedinger, J. R., Vogel, R. M., \& Foufoula-Georgiou, E. (1993). Frequency analysis of extreme events. In: Maidment, D.R. (ed.). Handbook of hydrology (pp: 18.1-18.66). New York, USA: McGraw-Hill.

UNESCO, Organización de las Naciones Unidas para la Educación, la Ciencia y la Cultura. (2010). Guía metodológica para la aplicación del análisis regional de frecuencia de sequías basado en L-momentos y 
resultados de aplicación en América Latina. Documentos Técnicos del PHI-LAC, No 27. Montevideo, Uruguay: Centro del Agua para Zonas Áridas y Semiáridas de América Latina y el Caribe.

Valiente, O. M. (2001). Sequía: definiciones, tipologías y métodos de cuantificación. Investigaciones Geográficas, 26, 59-80.

Velasco, I., Aparicio, J., Valdéz, J. B., Velázquez, J., \& Kim, T. W. (2004). Evaluación de índices de sequía en las cuencas de afluentes del río Bravo/Grande. Ingeniería Hidráulica en México, 19(3), 37-53.

Vich, A. I., Norte, F. A., \& Lauro, C. (2014). Análisis regional de frecuencias de caudales de ríos pertenecientes a cuencas con nacientes en la cordillera de los andes. Meteorológica, 39(1), 3-26.

Wallis, J. R., Schaefer, M. G., Barker, B. L., \& Taylor, G. H. (2007). Regional precipitation-frequency analysis and spatial mapping for 24hour and 2-hour durations for Washington State. Hydrology and Earth System Sciences, 11(1), 415-442.

Wilhite, D. A. (2000). Drought as a natural hazard: Concepts and definitions. In: Wilhite, D. A. (ed.). Drought: A global assessment. Vol. I (pp. 3-18). New York, USA: Routledge.

Wilhite, D. A., \& Glantz, M. H. (1985). Understanding the drought phenomenon: The role of definitions. Water International, 10, 111120. 